\title{
First General Zagreb Index of Generalized F-sum Graphs
}

\author{
H. M. Awais, ${ }^{1}$ Muhammad Javaid $\mathbb{D}^{1},{ }^{1}$ and Akbar Ali $\mathbb{D}^{2}$ \\ ${ }^{1}$ Department of Mathematics, School of Science, University of Management and Technology, Lahore, Pakistan \\ ${ }^{2}$ Department of Mathematics, Faculty of Science, University of Ha'il, Ha'il, Saudi Arabia
}

Correspondence should be addressed to Akbar Ali; akbarali.maths@gmail.com

Received 14 May 2020; Accepted 10 November 2020; Published 9 December 2020

Academic Editor: Luisa Di Paola

Copyright $\odot 2020$ H. M. Awais et al. This is an open access article distributed under the Creative Commons Attribution License, which permits unrestricted use, distribution, and reproduction in any medium, provided the original work is properly cited.

The first general Zagreb (FGZ) index (also known as the general zeroth-order Randić index) of a graph $G$ can be defined as $M^{\gamma}(G)=\sum_{u v \in E(G)}\left[d_{G}^{c-1}(u)+d_{G}^{c-1}(v)\right]$, where $\gamma$ is a real number. As $M^{\gamma}(G)$ is equal to the order and size of $G$ when $\gamma=0$ and $\gamma=1$, respectively, $\gamma$ is usually assumed to be different from 0 to 1 . In this paper, for every integer $\gamma \geq 2$, the FGZ index $M^{\gamma}$ is computed for the generalized F-sums graphs which are obtained by applying the different operations of subdivision and Cartesian product. The obtained results can be considered as the generalizations of the results appeared in (IEEE Access; 7 (2019) 47494-47502) and (IEEE Access 7 (2019) 105479-105488).

\section{Introduction}

Graph theory concepts are being utilized to model and study the several problems in different fields of science, including chemistry and computer science. A topological index (TI) of a (molecular) graph is a numeric quantity that remained unchanged under graph isomorphism [1,2]. Many topological indices have found applications in chemistry, especially in the quantitative structure-activity/property relationships studies; for detail, see [3-13].

Wiener index is the first TI introduced by Harry Wiener in 1947, when he was working on the boiling point of paraffin [14]. In 1972, Trinajstić and Gutman [15] obtained a formula concerning the total energy of $\pi$ electrons of molecules where the sum of square of valences of the vertices of a molecular structure was appeared. This sum is nowadays known as the first Zagreb index. In this paper, we are concerned with a generalized version of the first Zagreb index, known as the general first Zagreb index as well as the general zeroth-order Randić index.

There are several operations in graph theory such as product, complement, addition, switching, subdivision, and deletion. In many cases, graph operations may be helpful in finding graph quantities of more complicated graphs by considering the less complicated ones. In chemical graph theory, by using different graph operations, one can develop large molecular structures from the simple and basic structures. Recently, many classes of molecular structures are studied with the assistance of graph operations.

In 2007, Yan et al. [6] listed the five subdivision operations with the help of their vertices and edges. They also discussed the different features of Wiener index of graphs under these operations. After that, Eliasi and Taeri [16] introduced the $F_{1}$-sum graphs $\Gamma_{1+F_{1}} \Gamma_{2}$ with the assistance of Cartesian product on graphs $F_{1}\left(\Gamma_{1}\right)$ and $\Gamma_{2}$, where $F_{1}\left(\Gamma_{1}\right)$ is obtained by applying the subdivision operations $S_{1}, R_{1}, Q_{1}$, and $T_{1}$. They also defined the Wiener indices of these resulting graphs $\Gamma_{1+S_{1}} \Gamma_{2}, \Gamma_{1+R_{1}} \Gamma_{2}, \Gamma_{1+Q_{1}} \Gamma_{2}$, and $\Gamma_{1+T_{1}} \Gamma_{2}$. Later on, Deng et al. [17] calculated the 1st and 2nd Zagreb topological indices, and Imran and Akhtar [18] calculated the forgotten topological index of the $F_{1}$-sums graph. In 2019, Liu et al. [19] computed the first general Zagreb index of $F_{1}$-sums graphs.

Recently, Liu et al. [20] introduced the generalized version of the aforesaid subdivided operations of graphs denoted by $S_{k}, R_{k}, Q_{k}$, and $T_{k}$, where $k \geq 1$ is counting number. They also defined the generalized F-sums graphs using these generalized operations and calculated their 1st and 2 nd Zagreb indices. In the present work, we compute the 1 st general Zagreb index of the generalized $F$-sums graphs 
$\Gamma_{1+F_{k}} \Gamma_{2}$ for $F_{k} \in\left\{S_{k}, R_{k}, Q_{k}, T_{k}\right\}$. The remaining work is arranged as follows: Section 2 contains some basic definitions, Section 3 contains the key outcomes, and Section 4 contains the some particular applications. Conclusions of the obtained results are presented in Section 5.

\section{Preliminaries}

Let $\Gamma=(V(\Gamma), E(\Gamma))$ be a simple graph having $|V(\Gamma)|$ the order and $|E(\Gamma)|$ the size of a graph, where $V(\Gamma)$ is considered as node set and $E(\Gamma) \subseteq V(\Gamma) \times V(\Gamma)$ is a bond set. Every vertex is considered as an atom in a graph, and bonding within the two atoms is known as edge. The valency or degree of any node is the number of total edges which are incident to the node. Now, few useful TI's are explained given below:

Definition 1. If $\Gamma$ be a connected graph, then the 1 st and 2 nd Zagreb topological indices as

$$
\begin{aligned}
& M_{1}(\Gamma)=\sum_{u v \in V(\Gamma)}\left[d_{\Gamma}(u)+d_{\Gamma}(v)\right], \\
& M_{2}(\Gamma)=\sum_{u v \in V(\Gamma)}\left[d_{\Gamma}(u) d_{\Gamma}(v)\right] .
\end{aligned}
$$

These two descriptors of the graph were introduced by Trinajsti and Gutman [15]. Such type of TI's have been utilized to discuss the QSAR/QSPR of the different chemical structures such as chirality, complexity, hetero-system, ZEisomers, $\pi$ electron energy, and branching $[9,10]$.

Definition 2. If $R$ is the real number, $\gamma \in R-\{0,1\}$, and $\Gamma$ be a connected graph, so the 1st general Zagreb topological index is given as

$$
M^{\gamma}(\Gamma)=\sum_{u v \in E(\Gamma)}\left[d_{\Gamma}^{\gamma-1}(u)+d_{\Gamma}^{\gamma-1}(v)\right] .
$$

Definition 3. If $R$ is the real number, $\gamma \in R$, and $\Gamma$ be a connected graph, so the general Randic is given as

$$
R_{\gamma}(\Gamma)=\sum_{u v \in E(\Gamma)}\left[d_{G}(u) d_{(v)}\right]^{\gamma},
$$

where $R_{-(1 / 2)}$ is considered as the classical Randic connectivity topological index.

The generalized F-sums graph is defined in [20] as follows:

(i) $S_{k}(G)$ graph is obtained by inserting $k$ vertices in each edge of $G$.

(ii) $R_{k}(G)$ is obtained from $S_{k}(G)$ by joining the old vertices which are adjacent $G$.

(iii) $Q_{k}(G)$ is obtained from $S_{k}(G)$ by joining the new vertices lying on edge to the corresponding new vertices of other edge, if these edges have some common vertex in $G$.

(iv) $T_{k}(G)$ is union of $R_{k}(G)$ and $Q_{k}(G)$ graphs. For further details, see Figure 1.

Definition 4. If $\Gamma_{1} \& \Gamma_{2}$ be two connected molecular structures, $F_{k} \in\left\{S_{k}, R_{k}, Q_{k}, T_{k}\right\}$ and $F_{k}\left(\Gamma_{1}\right)$ be a structure obtained after using $F_{k}$ on $\Gamma_{1}$ with bonds (edges) $E\left(F_{k}\left(\Gamma_{1}\right)\right)$ and nodes (vertices) $V\left(F_{k}\left(\Gamma_{1}\right)\right)$. So, the generalized $F$-sums graph $\left(\Gamma_{1+F_{k}} \Gamma_{2}\right)$ is a structure with nodes:

$$
\begin{aligned}
V\left(\Gamma_{1+F_{k}} \Gamma_{2}\right) & =V\left(F_{k}\left(\Gamma_{1}\right)\right) \times V\left(\Gamma_{2}\right), \\
& =\left(V\left(\Gamma_{1}\right) \cup E\left(\Gamma_{1}\right)\right) \times V\left(\Gamma_{2}\right),
\end{aligned}
$$

in such a way two nodes $\left(a_{1}, b_{1}\right) \&\left(a_{2}, b_{2}\right)$ of $V\left(\Gamma_{1+F_{k}} \Gamma_{2}\right)$ are adjacent if $\left[a_{1}=a_{2} \in V\left(\Gamma_{1}\right) \&\left(b_{1}, b_{2}\right) \in E\left(\Gamma_{2}\right)\right]$ or $\left[b_{1}=b_{2} \in V\left(\Gamma_{2}\right) \&\left(a_{1}, a_{2}\right) \in E\left(F_{k}\left(\Gamma_{1}\right)\right)\right]$. For more details, see Figures 2 and 3.

Lemma 1. For $F_{k} \in\left\{S_{k}, R_{k}, Q_{k}, T_{k}\right\}$ and $(x, y) \in \Gamma_{1}+{ }_{F_{k}} \Gamma_{2}$, the degree of $(x, y)$ in $\Gamma_{1}+{ }_{F_{k}} \Gamma_{2}$ is

$$
\begin{aligned}
\text { (i) } d\left(d_{\Gamma_{1}+S_{k} \Gamma_{2}}(x, y)\right) & = \begin{cases}d_{\Gamma_{1}}(x)+d_{\Gamma_{2}}(y), & \text { if } x \in V\left(\Gamma_{1}\right) \wedge y \in V\left(\Gamma_{2}\right), \\
2, & \text { if } x \in V\left(S_{k}\left(\Gamma_{1}\right)\right)-V\left(\Gamma_{1}\right) \wedge y \in V\left(\Gamma_{2}\right),\end{cases} \\
\text { (ii) } d\left(d_{\Gamma_{1}+R_{k} \Gamma_{2}}(x, y)\right) & = \begin{cases}d_{R_{k}\left(\Gamma_{1}\right)}(x)+d_{\Gamma_{2}}(y), & \text { if } x \in V\left(\Gamma_{1}\right) \wedge y \in V\left(\Gamma_{2}\right), \\
2, & \text { if } x \in V\left(S_{k}\left(\Gamma_{1}\right)\right)-V\left(\Gamma_{1}\right) \wedge y \in V\left(\Gamma_{2}\right),\end{cases} \\
\text { (iii) } d\left(d_{\Gamma_{1}+Q_{k} \Gamma_{2}}(x, y)\right) & = \begin{cases}d_{\Gamma_{1}}(x)+d_{\Gamma_{2}}(y), & \text { if } x \in V\left(\Gamma_{1}\right) \wedge y \in V\left(\Gamma_{2}\right), \\
d_{Q_{k}\left(\Gamma_{1}\right)}(x), & \text { if } x \in V\left(Q_{k}\left(\Gamma_{1}\right)\right)-V\left(\Gamma_{1}\right) \wedge y \in V\left(\Gamma_{2}\right),\end{cases} \\
\text { (iv) } d\left(d_{\Gamma_{1}+T_{k} \Gamma_{2}}(x, y)\right) & = \begin{cases}d_{T_{k}\left(\Gamma_{1}\right)}(x)+d_{\Gamma_{2}}(y), & \text { if } x \in V\left(\Gamma_{1}\right) \wedge y \in V\left(\Gamma_{2}\right), \\
d_{T_{k}\left(\Gamma_{1}\right)}(x), & \text { if } x \in V\left(T_{k}\left(\Gamma_{1}\right)\right)-V\left(\Gamma_{1}\right) \wedge y \in V\left(\Gamma_{2}\right) .\end{cases}
\end{aligned}
$$




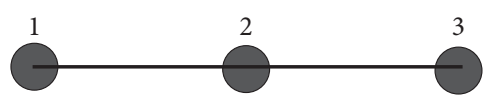

(a)

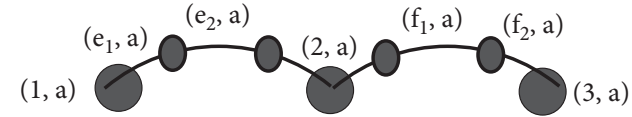

(b)
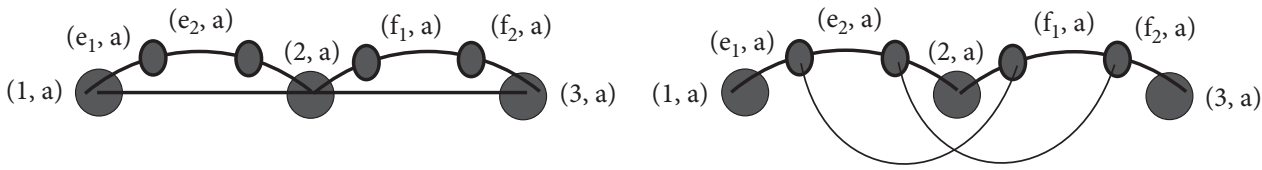

(c)

(d)

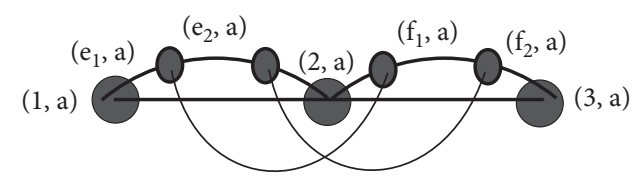

(e)

Figure 1: (a) $\Gamma$, (b). $S_{2}(\Gamma)$, (c) $R_{2}(\Gamma),(\mathrm{d}) Q_{2}(\Gamma)$, and (e) $T_{2}(\Gamma)$.
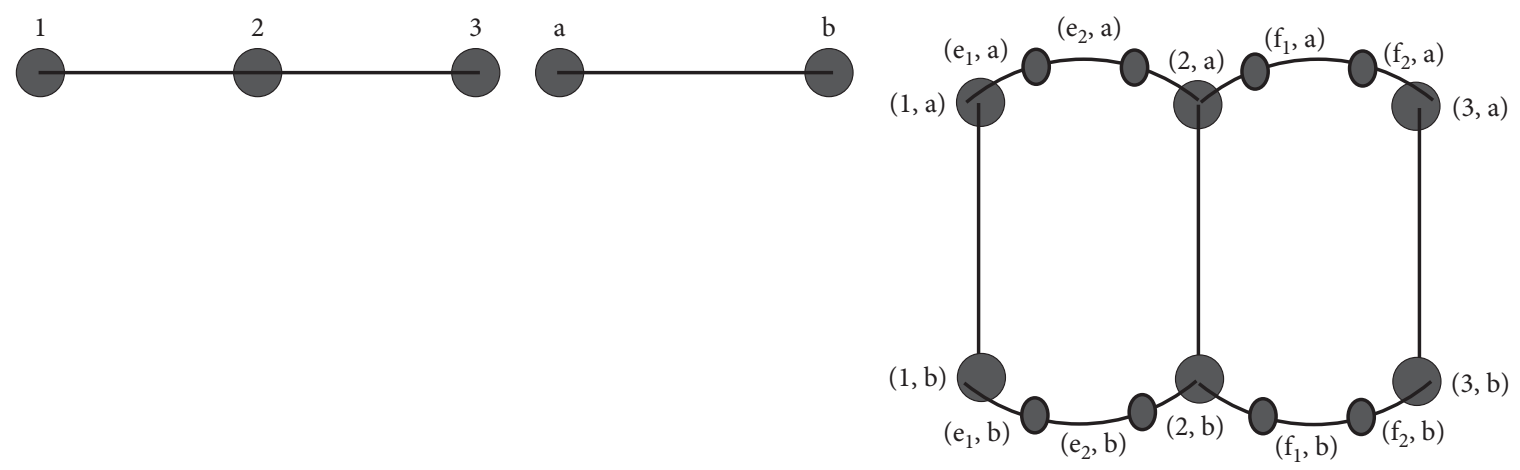

(a)

(b)

(c)

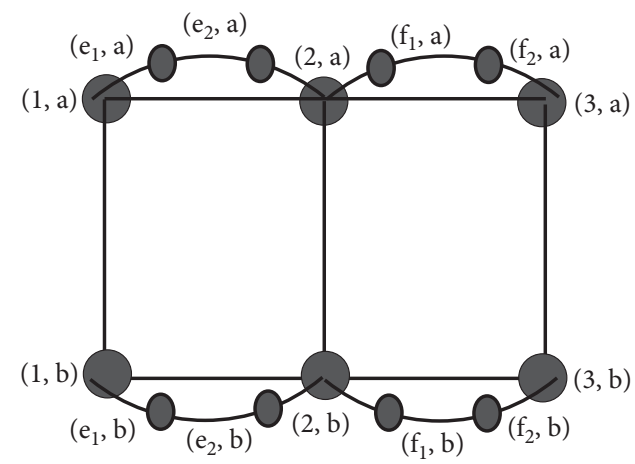

(d)

Figure 2: (a) $\Gamma_{1} \cong P_{3}$. (b) $\Gamma_{2} \cong P_{2}$. (c) $\Gamma_{1+S_{2}} \Gamma_{2}$. (d) $\Gamma_{1+R_{2}} \Gamma_{2}$.

\section{Main Results}

The main results of FGZ index of the generalized F-sum graphs are presented in this section.

Theorem 1. Let $\Gamma_{1}$ and $\Gamma_{2}$ be two simple graphs and $\gamma \in N-\{0,1\}$. The FGZ index of the generalized S-sum graph $\Gamma_{1}+S_{k} \Gamma_{2}$ is

$$
\begin{aligned}
M^{\gamma}\left(\Gamma_{1}+S_{k} \Gamma_{2}\right)= & \sum_{i=0}^{\alpha}\left(\begin{array}{c}
\alpha \\
i
\end{array}\right)\left(M_{\Gamma_{1}}^{\alpha-i}\right)\left(M_{\Gamma_{2}}^{i+1}\right)+n_{\Gamma_{2}} M_{S_{k}\left(\Gamma_{1}\right)}^{\gamma} \\
& +\sum_{i=1}^{\alpha}\left(\begin{array}{c}
\alpha \\
i
\end{array}\right) M_{\Gamma_{2}}^{i} M_{\Gamma_{1}}^{\gamma-i}+2^{\alpha+1}(k-1) n_{\Gamma_{2}} e_{\Gamma_{1}},
\end{aligned}
$$

where $N$ is the set of natural numbers and $\alpha=\gamma-1$. 


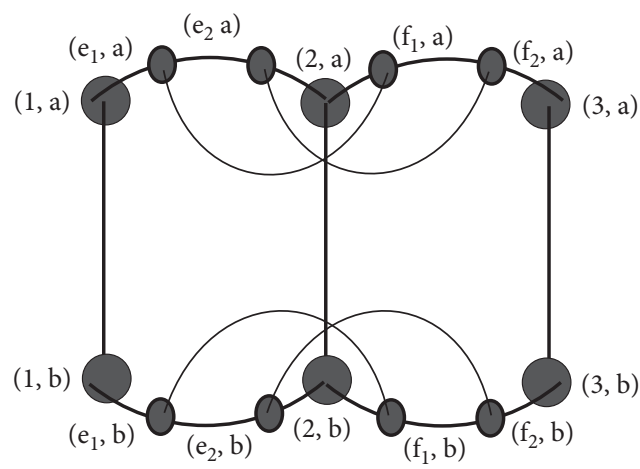

(a)

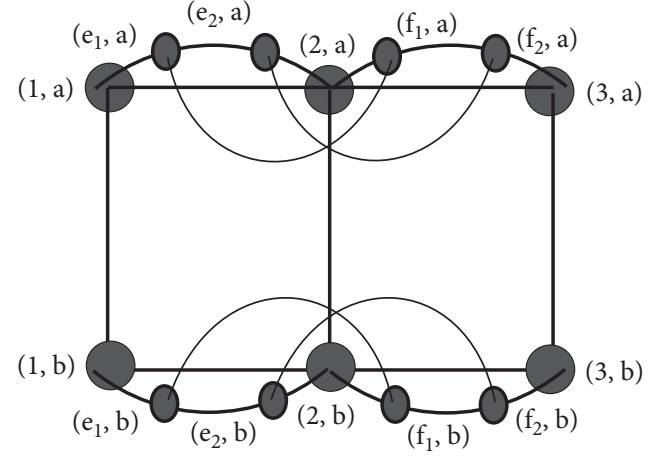

(b)

Figure 3: (a) $\Gamma_{1+Q_{2}} \Gamma_{2}$. (b) $\Gamma_{1+T_{2}} \Gamma_{2}$.

Proof. Let

$$
M^{\gamma}\left(\Gamma_{1}+{ }_{S_{k}} \Gamma_{2}\right)=\sum_{(a, b) \in V\left(\Gamma_{1}+s_{k} \Gamma_{2}\right)} d_{\Gamma_{1}+s_{k} \Gamma_{2}}^{\gamma}(a, b) .
$$

$$
\begin{aligned}
M^{\gamma}\left(\Gamma_{1}+s_{k} \Gamma_{2}\right)= & \sum_{(a, b)(c, d) \in E\left(\Gamma_{1}+s_{k} \Gamma_{2}\right)}\left[d_{\Gamma_{1}+s_{k} \Gamma_{2}}^{\alpha}(a, b)+d_{\Gamma_{1}+s_{k} \Gamma_{2}}^{\alpha}(c, d)\right] \\
= & \sum_{a \in V\left(\Gamma_{1}\right)} \sum_{b d \in E\left(\Gamma_{2}\right)}\left[d_{\Gamma_{1}+s_{k} \Gamma_{2}}^{\alpha}(a, b)+d_{\Gamma_{1}+s_{k} \Gamma_{2}}^{\alpha}(a, d)\right]+\sum_{b \in V\left(\Gamma_{2}\right)} \sum_{a c \in E\left(S_{k}\left(\Gamma_{1}\right)\right)}\left[d_{\Gamma_{1}+s_{k} \Gamma_{2}}^{\alpha}(a, b)+d_{\Gamma_{1}+s_{k} \Gamma_{2}}^{\alpha}(b, c)\right] \\
= & \sum_{a \in V\left(\Gamma_{1}\right)} \sum_{b d \in E\left(\Gamma_{2}\right)}\left[d_{\Gamma_{1}+s_{k} \Gamma_{2}}^{\alpha}(a, b)+d_{\Gamma_{1}+s_{k} \Gamma_{2}}^{\alpha}(a, d)\right]+\sum_{b \in V\left(\Gamma_{2}\right) a c \in E\left(s_{k}\left(\Gamma_{1}\right)\right) a \in V\left(\Gamma_{1}\right), c \in V\left(s_{k}\left(\Gamma_{1}\right)\right)-V\left(\Gamma_{1}\right)}\left[d_{\Gamma_{1}+s_{k} \Gamma_{2}}^{\alpha}(a, b)+d_{\Gamma_{1}+s_{k} \Gamma_{2}}^{\alpha}(b, c)\right] \\
& +\sum_{b \in V\left(\Gamma_{2}\right)}\left[d_{a c \in E\left(S_{k}\left(\Gamma_{1}\right)\right) a, c \in V\left(s_{k}\left(\Gamma_{1}\right)\right)-V\left(\Gamma_{1}\right)}^{\alpha}(a, b)+d_{\Gamma_{1}+s_{k} \Gamma_{2}}^{\alpha}(b, c)\right] .
\end{aligned}
$$

For every vertex $a \in V\left(\Gamma_{1}\right)$ and edge $b d \in E\left(\Gamma_{2}\right)$, then 1 st term of (8) will be

$$
\begin{aligned}
& \sum_{a \in V\left(\Gamma_{1}\right)} \sum_{b d \in E\left(\Gamma_{2}\right)}\left[d_{\Gamma_{1}+S_{k} \Gamma_{2}}^{\alpha}(a, b)+d_{\Gamma_{1}+s_{k} \Gamma_{2}}^{\alpha}(a, d)\right] \\
& =\sum_{a \in V\left(\Gamma_{1}\right)} \sum_{b d \in E\left(\Gamma_{2}\right)}\left[\sum_{i=0}^{\alpha}\left(\begin{array}{c}
\alpha \\
i
\end{array}\right) d_{\Gamma_{1}}^{\alpha-i}(a) d_{\Gamma_{2}}^{i}(b)+\sum_{i=0}^{\alpha}\left(\begin{array}{c}
\alpha \\
i
\end{array}\right) d_{\Gamma_{1}}^{\alpha-i}(a) d_{\Gamma_{2}}^{i}(d)\right] \\
& =\sum_{a \in V\left(\Gamma_{1}\right)} \sum_{b d \in E\left(\Gamma_{2}\right)}\left[\sum_{i=0}^{\alpha}\left(\begin{array}{c}
\alpha \\
i
\end{array}\right) d_{\Gamma_{1}}^{\alpha-i}(a)\left[d_{\Gamma_{2}}^{i}(b)+d_{\Gamma_{2}}^{i}(d)\right]\right] \\
& =\sum_{a \in V\left(\Gamma_{1}\right)}\left[\sum_{i=0}^{\alpha}\left(\begin{array}{c}
\alpha \\
i
\end{array}\right) d_{\Gamma_{1}}^{\alpha-i}(a)\right]\left[\sum_{b d \in E\left(\Gamma_{2}\right)}\left[d_{\Gamma_{2}}^{i}(b)+d_{\Gamma_{2}}^{i}(d)\right]\right] \\
& \left.=\sum_{a \in V\left(\Gamma_{1}\right)}\left[\sum_{i=0}^{\alpha}\left(\begin{array}{c}
\alpha \\
i
\end{array}\right) d_{\Gamma_{1}}^{\alpha-i}(a)\right]\left(M_{\Gamma_{2}}^{i+1}\right)\right] \\
& =\sum_{i=0}^{\alpha}\left(\begin{array}{c}
\alpha \\
i
\end{array}\right)\left(M_{\Gamma_{1}}^{\alpha-i}\right)\left(M_{\Gamma_{2}}^{i+1}\right) .
\end{aligned}
$$


Since $\left|E\left(S_{k}\left(\Gamma_{1}\right)\right)\right|=2\left|E\left(\Gamma_{1}\right)\right|$. So, for every $b \in V\left(\Gamma_{2}\right)$ and $a c \in E\left(S_{k}\left(\Gamma_{1}\right)\right)$ with $a \in V\left(\Gamma_{1}\right)$, and $c \in V\left(S_{k}\left(\Gamma_{1}\right)\right)-V\left(\Gamma_{1}\right)$; then the 2 nd term of $(8)$ is

$$
\begin{aligned}
& \sum_{b \in V\left(\Gamma_{2}\right)} \sum_{a c \in E\left(s_{k}\left(\Gamma_{1}\right)\right) a \in V\left(\Gamma_{1}\right), c \in V\left(s_{k}\left(\Gamma_{1}\right)\right)-V\left(\Gamma_{1}\right)}\left[d_{\Gamma_{1}+s_{k} \Gamma_{2}}^{\alpha}(a, b)+d_{\Gamma_{1}+s_{k} \Gamma_{2}}^{\alpha}(b, c)\right] \\
& =\sum_{b \in V\left(\Gamma_{2}\right)} \sum_{a c \in E\left(s_{k}\left(\Gamma_{1}\right)\right)}\left[d_{\Gamma_{1}+s_{k} \Gamma_{2}}^{\alpha}\right](a, b)+d_{\Gamma_{1}+s_{k} \Gamma_{2}}^{\alpha}(b, c) \\
& =\sum_{b \in V\left(\Gamma_{2}\right)} \sum_{a c \in E\left(S_{k}\left(\Gamma_{1}\right)\right)}\left[\sum_{i=0}^{\alpha}\left(\begin{array}{c}
\alpha \\
i
\end{array}\right) d_{S_{k}\left(\Gamma_{1}\right)}^{\alpha-i}(a) d_{\Gamma_{2}}^{i}(b)+d_{S_{k}\left(\Gamma_{1}\right)}^{\alpha}(c)\right] \\
& =\sum_{b \in V\left(\Gamma_{2}\right)} \sum_{a c \in E\left(S_{k}\left(\Gamma_{1}\right)\right)}\left[d_{S_{k}\left(\Gamma_{1}\right)}^{\alpha}(a)+\sum_{i=0}^{\alpha}\left(\begin{array}{c}
\alpha \\
i
\end{array}\right) d_{S_{k}\left(\Gamma_{1}\right)}^{\alpha-i}(a) \cdot d_{\Gamma_{2}}^{i}(b)+d_{S_{k}\left(\Gamma_{1}\right)}^{\alpha}(c)\right] \\
& =\sum_{b \in V\left(\Gamma_{2}\right)} \sum_{a c \in E\left(S_{k}\left(\Gamma_{1}\right)\right)}\left[d_{S_{k}\left(\Gamma_{1}\right)}^{\alpha}(a)+d_{S_{k}\left(\Gamma_{1}\right)}^{\alpha}(c)+\sum_{i=1}^{\alpha}\left(\begin{array}{c}
\alpha \\
i
\end{array}\right) d_{S_{k}\left(\Gamma_{1}\right)}^{\alpha-i}(a) d_{\Gamma_{2}}^{i}(b)\right] \\
& =\sum_{b \in V\left(\Gamma_{2}\right)} \sum_{a c \in E\left(S_{k}\left(\Gamma_{1}\right)\right)}\left[d_{S_{k}\left(\Gamma_{1}\right)}^{\alpha}(a)+d_{S_{k}\left(\Gamma_{1}\right)}^{\alpha}(c)\right]+\sum_{b \in V\left(\Gamma_{2}\right)} \sum_{a c \in E\left(S_{k}\left(\Gamma_{1}\right)\right)}\left[\sum_{i=1}^{\alpha}\left(\begin{array}{c}
\alpha \\
i
\end{array}\right) d_{S_{k}\left(\Gamma_{1}\right)}^{\alpha-i}(a) d_{\Gamma_{2}}^{i}(b)\right] \\
& =\sum_{b \in V\left(\Gamma_{2}\right)}\left[M_{S_{k}\left(\Gamma_{1}\right)}^{\alpha+1}\right]+\sum_{i=1}^{\alpha}\left(\begin{array}{c}
\alpha \\
i
\end{array}\right)\left[M_{\Gamma_{2}}^{i}\right]\left[M_{\Gamma_{1}}^{\gamma-i}\right] \\
& =n_{\Gamma_{2}}\left[M_{S_{k}\left(\Gamma_{1}\right)}^{\gamma}\right]+\sum_{i=1}^{\alpha}\left(\begin{array}{c}
\alpha \\
i
\end{array}\right)\left[M_{\Gamma_{2}}^{i}\right]\left[M_{\Gamma_{1}}^{\gamma-i}\right] \text {, }
\end{aligned}
$$

and the 3rd term of equation (8) will be

$$
\sum_{b \varepsilon V\left(\Gamma_{2}\right)} \sum_{a c \varepsilon E\left(S_{k}\left(\Gamma_{1}\right)\right) a, c \varepsilon V\left(S_{k}\left(\Gamma_{1}\right)\right)-V\left(\Gamma_{1}\right)}\left[d_{\Gamma_{1}+s_{k} \Gamma_{2}}^{\alpha}(a, b)+d_{\Gamma_{1}+s_{k} \Gamma_{2}}^{\alpha}(c, b)\right]=\sum_{b \varepsilon V\left(\Gamma_{2}\right) a c \varepsilon E\left(s_{k}\left(\Gamma_{1}\right)\right) a, c \varepsilon V\left(S_{k}\left(\Gamma_{1}\right)\right)-V\left(\Gamma_{1}\right)}\left[2^{\alpha}+2^{\alpha}\right] .
$$

Since in this case $\left|E\left(S_{k}\left(\Gamma_{1}\right)\right)\right|=(k-1) \mid e_{\Gamma_{1}}$, we have

By using (9), (10), \& (12) in (8), we get

$$
=2^{\alpha+1}(k-1) n_{\Gamma_{2}} e_{\Gamma_{1}} \text {. }
$$

$$
M^{\gamma}\left(\Gamma_{1}+S_{k} \Gamma_{2}\right)=\sum_{i=0}^{\alpha}\left(\begin{array}{c}
\alpha \\
i
\end{array}\right)\left(M_{\Gamma_{1}}^{\alpha-i}\right)\left(M_{\Gamma_{2}}^{i+1}\right)+n_{\Gamma_{2}} M_{S_{k}\left(\Gamma_{1}\right)}^{\beta}+\sum_{i=1}^{\alpha}\left(\begin{array}{c}
\alpha \\
i
\end{array}\right) M_{\Gamma_{2}}^{i} M_{\Gamma_{1}}^{\gamma-i}+2^{\alpha+1}(k-1) n_{\Gamma_{2}} e_{\Gamma_{1}} .
$$

Theorem 2. Let $\Gamma_{1}$ and $\Gamma_{2}$ be two simple graphs and $\gamma \in N-\{0,1\}$. The FGZ index of the generalized R-sum $\Gamma_{1}+{ }_{R_{k}} \Gamma_{2}$ graph is

$$
\begin{aligned}
M^{\gamma}\left(\Gamma_{1}+{ }_{R_{k}} \Gamma_{2}\right)= & \sum_{i=0}^{\alpha}\left(\begin{array}{c}
\alpha \\
i
\end{array}\right) 2^{\alpha-i} M_{\Gamma_{1}}^{\alpha-i} M_{\Gamma_{2}}^{i+1}+2 \sum_{i=0}^{\alpha}\left(\begin{array}{c}
\alpha \\
i
\end{array}\right) 2^{\alpha-i} M_{\Gamma_{1}}^{\gamma-i} M_{\Gamma_{2}}^{i} \\
& +2^{\alpha} e_{\Gamma_{1}} n_{\Gamma_{2}}+2^{\alpha+1}(k-1) n_{\Gamma_{2}} e_{\Gamma_{1}},
\end{aligned}
$$

where $N$ is the set of natural numbers and $\alpha=\gamma-1$.

Proof. Then by definition, we have,

$$
M^{\gamma}\left(\Gamma_{1}+R_{k} \Gamma_{2}\right)=\sum_{(a, b) \in\left(V\left(\Gamma_{1}+R_{k} \Gamma_{2}\right)\right)} d_{\Gamma_{1}+R_{k} \Gamma_{2}}^{\gamma}(a, b) .
$$

For $\alpha=\gamma-1$, the above equation is consider as 


$$
\begin{aligned}
M^{\gamma}\left(\Gamma_{1}+{ }_{R_{k}} \Gamma_{2}\right)= & \sum_{(a, b)(c, d) \in\left(E\left(\Gamma_{1}+R_{k} \Gamma_{2}\right)\right)}\left[d_{\Gamma_{1}+R_{k} \Gamma_{2}}^{\alpha}(a, b)+d_{\Gamma_{1}+R_{k} \Gamma_{2}}^{\alpha}(c, d)\right] \\
= & \sum_{a \in V\left(\Gamma_{1}\right)} \sum_{b d \in\left(E\left(\Gamma_{2}\right)\right)}\left[d_{\Gamma_{1}+R_{k} \Gamma_{2}}^{\alpha}(a, b)+d_{\Gamma_{1}+R_{k} \Gamma_{2}}^{\alpha}(a, d)\right]+\sum_{b \in V\left(\Gamma_{2}\right) a c \in\left(E\left(R_{k}\left(\Gamma_{1}\right)\right)\right)}\left[d_{\Gamma_{1}+R_{k} \Gamma_{2}}^{\alpha}(a, b)+d_{\Gamma_{1}+R_{k} \Gamma_{2}}^{\alpha}(b, c)\right] \\
= & \sum_{a \in V\left(\Gamma_{1}\right) b d \in\left(E\left(\Gamma_{2}\right)\right)}\left[d_{\Gamma_{1}+R_{k} \Gamma_{2}}^{\alpha}(a, b)+d_{\Gamma_{1}+R_{k} \Gamma_{2}}^{\alpha}(a, d)\right]+\sum_{b \in V\left(\Gamma_{2}\right) a c \in\left(E\left(R_{k}\left(\Gamma_{1}\right)\right)\right) a, c \in V\left(\Gamma_{1}\right)}\left[d_{\Gamma_{1}+R_{k} \Gamma_{2}}^{\alpha}(a, b)+d_{\Gamma_{1}+R_{k} \Gamma_{2}}^{\alpha}(b, c)\right] \\
& +\sum_{b \in V\left(\Gamma_{2}\right) a c \in\left(E\left(R_{k}\left(\Gamma_{1}\right)\right)\right) a \in V\left(\Gamma_{1}\right) c \in V\left(R_{k}\left(\Gamma_{1}\right)\right)-V\left(\Gamma_{1}\right)}\left[d_{\Gamma_{1}+R_{k} \Gamma_{2}}^{\alpha}(a, b)+d_{\Gamma_{1}+R_{k} \Gamma_{2}}^{\alpha}(b, c)\right] \\
= & \sum_{a \in V\left(\Gamma_{1}\right) b d \in\left(E\left(\Gamma_{2}\right)\right)}\left[d_{\Gamma_{1}+R_{k} \Gamma_{2}}^{\alpha}(a, b)+d_{\Gamma_{1}+R_{k} \Gamma_{2}}^{\alpha}(a, d)\right]+\sum_{b \in V\left(\Gamma_{2}\right) a c \in\left(E\left(R_{k}\left(\Gamma_{1}\right)\right)\right) a, c \in V\left(\Gamma_{1}\right)}\left[d_{\Gamma_{1}+R_{R_{k}} \Gamma_{2}}^{\alpha}(a, b)+d_{\Gamma_{1}+R_{k} \Gamma_{2}}^{\alpha}(b, c)\right] \\
& +\sum_{b \in V\left(\Gamma_{2}\right) a c \in\left(E\left(R_{k}\left(\Gamma_{1}\right)\right)\right) a \in V\left(\Gamma_{1}\right) c \in V\left(R_{k}\left(\Gamma_{1}\right)\right)-V\left(\Gamma_{1}\right)}\left[d_{\Gamma_{1}+R_{k} \Gamma_{2}}^{\alpha}(a, b)+d_{\Gamma_{1}+R_{k} \Gamma_{2}}^{\alpha}(b, c)\right] \sum_{b \varepsilon V\left(\Gamma_{2}\right)} \\
& \cdot \sum_{a c \varepsilon E\left(R_{k}\left(\Gamma_{1}\right)\right) a, c \varepsilon V\left(R_{k}\left(\Gamma_{1}\right)\right)-V\left(\Gamma_{1}\right)}\left[d_{\Gamma_{1}+R_{k} \Gamma_{2}}^{\alpha}(a, b)+d_{\Gamma_{1}+R_{k} \Gamma_{2}}^{\alpha}(c, b)\right] .
\end{aligned}
$$

For every vertex $a \in V\left(\Gamma_{1}\right)$ \& edge $b d \in E\left(\Gamma_{2}\right)$, then the 1 st term of (16) is

$$
\begin{aligned}
& \sum_{a \in V\left(\Gamma_{1}\right)} \sum_{b d \in\left(E\left(\Gamma_{2}\right)\right)}\left[d_{\Gamma_{1}+R_{k} \Gamma_{2}}^{\alpha}(a, b)+d_{\Gamma_{1}+R_{k} \Gamma_{2}}^{\alpha}(a, d)\right]=\sum_{a \in V\left(\Gamma_{1}\right) b d \in\left(E\left(\Gamma_{2}\right)\right)}\left[\left[\sum_{i=0}^{\alpha}\left(\begin{array}{c}
\alpha \\
i
\end{array}\right) d_{R_{k}\left(\Gamma_{1}\right)}^{\alpha-i}(a) \cdot d_{\Gamma_{2}}^{i}(b)\right]+\left[\sum_{i=0}^{\alpha}\left(\begin{array}{c}
\alpha \\
i
\end{array}\right) d_{R_{k}\left(\Gamma_{1}\right)}^{\alpha-i}(a) \cdot d_{\Gamma_{2}}^{i}(d)\right]\right] \\
& =\sum_{i=0}^{\alpha}\left(\begin{array}{c}
\alpha \\
i
\end{array}\right) \sum_{a \in V\left(\Gamma_{1}\right)} d_{R_{k}\left(\Gamma_{1}\right)}^{\alpha-i}(a) \sum_{b d \in\left(E\left(\Gamma_{2}\right)\right)} d_{\Gamma_{2}}^{i}(b)+\sum_{i=0}^{\alpha}\left(\begin{array}{c}
\alpha \\
i
\end{array}\right) \sum_{a \in V\left(\Gamma_{1}\right)} d_{R_{k}\left(\Gamma_{1}\right)}^{\alpha-i}(a) \\
& \cdot \sum_{b d \in\left(E\left(\Gamma_{2}\right)\right)} d_{\Gamma_{2}}^{i}(d) \\
& =\sum_{i=0}^{\alpha}\left(\begin{array}{c}
\alpha \\
i
\end{array}\right) \sum_{a \in V\left(\Gamma_{1}\right)}\left(2 d_{\Gamma_{1}}(a)\right)^{\alpha-i} \sum_{b d \in\left(E\left(\Gamma_{2}\right)\right)} d_{\Gamma_{2}}^{i}(b)+\sum_{i=0}^{\alpha}\left(\begin{array}{c}
\alpha \\
i
\end{array}\right) \sum_{a \in V\left(\Gamma_{1}\right)}\left(2 d_{\Gamma_{1}}(a)\right)^{\alpha-i} \\
& \sum_{b d \in\left(E\left(\Gamma_{2}\right)\right)} d_{\Gamma_{2}}^{i}(d) \\
& =\sum_{i=0}^{\alpha}\left(\begin{array}{c}
\alpha \\
i
\end{array}\right)(2)^{\alpha-i} M_{\Gamma_{1}}^{\alpha-i} \sum_{b d \in\left(E\left(\Gamma_{2}\right)\right)}\left[d_{\Gamma_{2}}^{i}(b)+d_{\Gamma_{2}}^{i}(d)\right] \\
& =\sum_{i=0}^{\alpha}\left(\begin{array}{c}
\alpha \\
i
\end{array}\right)(2)^{\alpha-i} M_{\Gamma_{1}}^{\alpha-i} M_{\Gamma_{2}}^{i+1} \text {. }
\end{aligned}
$$


For every vertex $b \in V\left(\Gamma_{2}\right) \&$ edge $a c \in E\left(R_{k}\right.$ $\left.\left(\Gamma_{1}\right)\right) a, c \in V\left(\Gamma_{1}\right)$, then the 2 nd term of (16) will be

$$
\begin{aligned}
& \sum_{b \in V\left(\Gamma_{2}\right) a c \in\left(E\left(R_{k}\left(\Gamma_{1}\right)\right)\right)}\left[d_{\Gamma_{1}+R_{k} \Gamma_{2}}^{\alpha}(a, b)+d_{\Gamma_{1}+R_{k} \Gamma_{2}}^{\alpha}(b, c)\right] \\
& =\sum_{b \in V\left(\Gamma_{2}\right) a c \in\left(E\left(R_{k}\left(\Gamma_{1}\right)\right)\right)}\left[\left[\sum_{i=0}^{\alpha}\left(\begin{array}{c}
\alpha \\
i
\end{array}\right) d_{R_{k}\left(\Gamma_{1}\right)}^{\alpha-i}(a) \cdot d_{\Gamma_{2}}^{i}(b)\right]+\left[\sum_{i=0}^{\alpha}\left(\begin{array}{c}
\alpha \\
i
\end{array}\right) d_{R_{k}\left(\Gamma_{1}\right)}^{\alpha-i}(c) \cdot d_{\Gamma_{2}}^{i}(b)\right]\right] \\
& =\sum_{b \in V\left(\Gamma_{2}\right) a c \in\left(E\left(R_{k}\left(\Gamma_{1}\right)\right)\right)}\left[\sum_{i=0}^{\alpha}\left(\begin{array}{c}
\alpha \\
i
\end{array}\right) d_{\Gamma_{2}}^{i}(b)\left[d_{R_{k}\left(\Gamma_{1}\right)}^{\alpha-i}(a)+d_{R_{k}\left(\Gamma_{1}\right)}^{\alpha-i}(c)\right]\right] \\
& =\sum_{a c \in\left(E\left(R_{k}\left(\Gamma_{1}\right)\right)\right) a, c \in V\left(\Gamma_{1}\right)}\left[\left[\sum_{i=0}^{\alpha}\left(\begin{array}{c}
\alpha \\
i
\end{array}\right)\left[\sum_{b \in V\left(\Gamma_{2}\right)} d_{\Gamma_{2}}^{i}(b)\left[d_{R_{k}\left(\Gamma_{1}\right)}^{\alpha-i}(a)+d_{R_{k}\left(\Gamma_{1}\right)}^{\alpha-i}(c)\right]\right]\right]\right. \\
& =\sum_{a c \in\left(E\left(R_{k}\left(\Gamma_{1}\right)\right)\right) a, c \in V\left(\Gamma_{1}\right)}\left[\left[\sum_{i=0}^{\alpha}\left(\begin{array}{c}
\alpha \\
i
\end{array}\right) M_{\Gamma_{2}}^{i}\left[\left(2 d_{\Gamma_{1}}(a)\right)^{\alpha-i}+\left(2 d_{\Gamma_{1}}(c)\right)^{\alpha-i}\right]\right]\right] \\
& =\sum_{a c \in\left(E\left(R_{k}\left(\Gamma_{1}\right)\right)\right) a, c \in V\left(\Gamma_{1}\right)} \sum_{i=0}^{\alpha}\left(\begin{array}{c}
\alpha \\
i
\end{array}\right) M_{\Gamma_{2}}^{i}\left[(2)^{\alpha-i}\left(d_{\Gamma_{1}}^{\alpha-i}(a)\right)+(2)^{\alpha-i}\left(d_{\Gamma_{1}}^{\alpha-i}(c)\right)\right] \\
& =\sum_{i=0}^{\alpha}\left(\begin{array}{c}
\alpha \\
i
\end{array}\right) M_{\Gamma_{2}}^{i} \sum_{a c \in\left(E\left(R_{k}\left(\Gamma_{1}\right)\right)\right) a, c \in V\left(\Gamma_{1}\right)}\left[(2)^{\alpha-i}\left(d_{\Gamma_{1}}^{\alpha-i}(a)\right)+(2)^{\alpha-i}\left(d_{\Gamma_{1}}^{\alpha-i}(c)\right)\right] \\
& =\sum_{i=0}^{\alpha}\left(\begin{array}{c}
\alpha \\
i
\end{array}\right) M_{\Gamma_{2}}^{i} \sum_{a c \in\left(E\left(R_{k}\left(\Gamma_{1}\right)\right)\right) a, c \in V\left(\Gamma_{1}\right)}\left[(2)^{\alpha-i}\left(d_{\Gamma_{1}}^{\alpha-i}(a)\right)+(2)^{\alpha-i}\left(d_{\Gamma_{1}}^{\alpha-i}(c)\right)\right] \\
& =\sum_{i=0}^{\alpha}\left(\begin{array}{c}
\alpha \\
i
\end{array}\right) M_{\Gamma_{2}}^{i}(2)^{\alpha-i} \sum_{a c \in\left(E\left(R_{k}\left(\Gamma_{1}\right)\right)\right) a, c \in V\left(\Gamma_{1}\right)}\left[d_{\Gamma_{1}}^{\alpha-i}(a)+d_{\Gamma_{1}}^{\alpha-i}(c)\right] \\
& =\sum_{i=0}^{\alpha}\left(\begin{array}{c}
\alpha \\
i
\end{array}\right) M_{\Gamma_{2}}^{i}(2)^{\alpha-1}\left[M_{\Gamma_{1}}^{\gamma-i}\right] \\
& =\sum_{i=0}^{\alpha}\left(\begin{array}{c}
\alpha \\
i
\end{array}\right) M_{\Gamma_{2}}^{i}(2)^{\alpha-1} M_{\Gamma_{1}}^{\gamma-i}
\end{aligned}
$$

For every vertex $b \in V\left(\Gamma_{2}\right) \& \quad$ edge $\quad a c \in E \quad d_{R_{k}\left(\Gamma_{1}\right)}(a)=2 d_{\Gamma_{1}}(a) \forall a \in V\left(\Gamma_{1}\right) \quad$ also $\quad d_{R_{k}\left(\Gamma_{1}\right)}(c)=2 \forall c$ $\left(R_{k}\left(\Gamma_{1}\right)\right) a \in V\left(\Gamma_{1}\right), c \in v\left(R_{k}\left(\Gamma_{1}\right)\right)-V\left(\Gamma_{1}\right)$. Since we have $\in V\left(R_{k}\left(\Gamma_{1}\right)\right)-V\left(\Gamma_{1}\right)$. So the 3 rd term of $(16)$ will be

$$
\begin{aligned}
& =\sum_{b \in V\left(\Gamma_{2}\right)} \sum_{a c \in\left(E\left(R_{k}\left(\Gamma_{1}\right)\right)\right) a \in V\left(\Gamma_{1}\right) c \in V\left(R_{k}\left(\Gamma_{1}\right)\right)-V\left(\Gamma_{1}\right)}\left[d_{\Gamma_{1}+_{R_{k}} \Gamma_{2}}^{\alpha}(a, b)+d_{\Gamma_{1}+_{R_{k}} \Gamma_{2}}^{\alpha}(b, c)\right] \\
& =\sum_{b \in V\left(\Gamma_{2}\right) a c \in\left(E\left(R_{k}\left(\Gamma_{1}\right)\right)\right) a \in V\left(\Gamma_{1}\right) c \in V\left(R_{k}\left(\Gamma_{1}\right)\right)-V\left(\Gamma_{1}\right)}\left[\sum_{i=0}^{\alpha}\left(\begin{array}{c}
\alpha \\
i
\end{array}\right) d_{R_{k}\left(\Gamma_{1}\right)}^{\alpha-i}(a) \cdot d_{\Gamma_{2}}^{i}(b)+d_{R_{k}\left(\Gamma_{1}\right)}^{\alpha}(c)\right],
\end{aligned}
$$


Here $d_{R_{k}\left(\Gamma_{1}\right)}^{\alpha}=2^{\alpha}$ and $d_{R_{k}\left(\Gamma_{1}\right)}^{\alpha-i}(a)=\left(2 d_{\Gamma_{1}}(a)\right)^{\alpha-i}$ :

$$
\begin{aligned}
= & \sum_{b \in V\left(\Gamma_{2}\right) a c \in\left(E\left(R_{k}\left(\Gamma_{1}\right)\right)\right)} \sum_{a \in V\left(\Gamma_{1}\right) c \in V\left(R_{k}\left(\Gamma_{1}\right)\right)-V\left(\Gamma_{1}\right)}\left[\sum_{i=0}^{\alpha}\left(\begin{array}{c}
\alpha \\
i
\end{array}\right)\left(2 d_{\Gamma_{1}}(a)\right)^{\alpha-i} d_{\Gamma_{2}}^{i}(b)+2^{\alpha}\right] \\
= & \sum_{b \in V\left(\Gamma_{2}\right) a c \in\left(E\left(R_{k}\left(\Gamma_{1}\right)\right)\right) a \in V\left(\Gamma_{1}\right) c \in V\left(R_{k}\left(\Gamma_{1}\right)\right)-V\left(\Gamma_{1}\right)}\left[\sum_{i=0}^{\alpha}\left(\begin{array}{c}
\alpha \\
i
\end{array}\right)(2)^{\alpha-i}\left(d_{\Gamma_{1}}(a)\right)^{\alpha-i} d_{\Gamma_{2}}^{i}(b)+2^{\alpha}\right] \\
= & \sum_{i=0}^{\alpha}\left(\begin{array}{c}
\alpha \\
i
\end{array}\right)\left[\sum_{b \in V\left(\Gamma_{2}\right) a c \in\left(E\left(R_{k}\left(\Gamma_{1}\right)\right)\right) a \in V\left(\Gamma_{1}\right) c \in V\left(R_{k}\left(\Gamma_{1}\right)\right)-V\left(\Gamma_{1}\right)}^{\alpha-i}\left(d_{\Gamma_{1}}^{\alpha-i}(a)\right) d_{\Gamma_{2}}^{i}(b)\right] \\
& +\left[\begin{array}{c}
\left.\sum_{b \in V\left(\Gamma_{2}\right) a c \in\left(E\left(R_{k}\left(\Gamma_{1}\right)\right)\right) a \in V\left(\Gamma_{1}\right) c \in V\left(R_{k}\left(\Gamma_{1}\right)\right)-V\left(\Gamma_{1}\right)}^{\alpha}\right] \\
=
\end{array} \sum_{i=0}^{\alpha}\left(\begin{array}{c}
\alpha \\
i
\end{array}\right)(2)^{\alpha-i} \cdot M_{\Gamma_{2}}^{i} \cdot M_{\Gamma_{1}}^{\gamma-i}+2^{\alpha} e_{\Gamma_{1}} n_{\Gamma_{2}}\right. \\
= & \sum_{i=0}^{\alpha}\left(\begin{array}{c}
\alpha \\
i
\end{array}\right)(2)^{\alpha-i} \cdot M_{\Gamma_{2}}^{i} \cdot M_{\Gamma_{1}}^{\gamma-i}+2^{\alpha} e_{\Gamma_{1}} n_{\Gamma_{2}},
\end{aligned}
$$

and the 4 th term of $(5)$ is

$$
\sum_{b \varepsilon V\left(\Gamma_{2}\right) a c \varepsilon E\left(R_{k}\left(\Gamma_{1}\right)\right) a, c \varepsilon V\left(R_{k}\left(\Gamma_{1}\right)\right)-V\left(\Gamma_{1}\right)}\left[d_{\Gamma_{1}+R_{k} \Gamma_{2}}^{\alpha}(a, b)+d_{\Gamma_{1}+R_{k} \Gamma_{2}}^{\alpha}(c, b)\right]=\sum_{b \varepsilon V\left(\Gamma_{2}\right) a c \varepsilon E\left(R_{k}\left(\Gamma_{1}\right)\right) a, c \varepsilon V\left(R_{k}\left(\Gamma_{1}\right)\right)-V\left(\Gamma_{1}\right)}\left[2^{\alpha}+2^{\alpha}\right] .
$$

Since in this case $\left|E\left(S_{k}\left(\Gamma_{1}\right)\right)\right|=(k-1) \mid e_{\Gamma_{1}}$, we have

Using (17), (18), (20), and (22) in (16), then we have

$$
=2^{\alpha+1}(k-1) n_{\Gamma_{2}} e_{\Gamma_{1}} \text {. }
$$

$$
M\left(\Gamma_{1}+{ }_{R_{k}} \Gamma_{2}\right)=\sum_{i=0}^{\alpha}\left(\begin{array}{c}
\alpha \\
i
\end{array}\right) 2^{\alpha-i} M_{\Gamma_{1}}^{\alpha-i} M_{\Gamma_{2}}^{i+1}+2\left[\sum_{i=0}^{\alpha}\left(\begin{array}{c}
\alpha \\
i
\end{array}\right) 2^{\alpha-i} M_{\Gamma_{1}}^{\gamma-i} M_{\Gamma_{2}}^{i}\right]+2^{\alpha} e_{\Gamma_{1}} n_{\Gamma_{2}}+2^{\alpha+1}(k-1) n_{\Gamma_{2}} e_{\Gamma_{1}}
$$

Theorem 3. Let $\Gamma_{1}$ and $\Gamma_{2}$ be two simple graphs and $\gamma \in N-\{0,1\}$. The FGZ index of the generalized Q-sum $\Gamma_{1}+{ }_{Q_{k}} \Gamma_{2}$ graph is

$$
\begin{aligned}
M^{\gamma}\left(\Gamma_{1}+{ }_{Q_{k}} \Gamma_{2}\right)= & \sum_{i=0}^{\alpha}\left(\begin{array}{c}
\alpha \\
i
\end{array}\right)\left(M_{\Gamma_{1}}^{\alpha-i}\right)\left(M_{\Gamma_{2}}^{i+1}\right)+\sum_{i=0}^{\alpha}\left(\begin{array}{c}
\alpha \\
i
\end{array}\right)\left(M_{\Gamma_{1}}^{\gamma-i}\right)\left(M_{\Gamma_{2}}^{i}\right)+2 n_{\Gamma_{2}} \sum_{i=0}^{\alpha}\left(\begin{array}{c}
\alpha \\
i
\end{array}\right)\left(d_{\Gamma_{1}}^{\alpha-i}(u) \cdot d_{\Gamma_{1}}^{i}(v)\right) \\
& +n_{\Gamma_{2}} \sum_{u v \in E\left(\Gamma_{1}\right) v w \in E\left(\Gamma_{1}\right)}\left[\left[\sum_{i=0}^{\alpha}\left(\begin{array}{c}
\alpha \\
i
\end{array}\right) d_{\Gamma_{1}}^{\alpha-i}(u) \cdot d_{\Gamma_{1}}^{i}(v)\right]+\left[\sum_{i=0}^{\alpha}\left(\begin{array}{c}
\alpha \\
i
\end{array}\right) d_{\Gamma_{1}}^{\alpha-i}(v) \cdot d_{\Gamma_{1}}^{i}(w)\right]\right] \\
& +2(k-1) n_{\Gamma_{2}} \sum_{u v \varepsilon E\left(\Gamma_{1}\right)}\left[d_{\Gamma_{1}}^{\alpha}(u)+d_{\Gamma_{1}}^{\alpha}(v)\right],
\end{aligned}
$$

where $N$ is the set of natural numbers and $\alpha=\gamma-1$.

Proof. Then by definition, we have 


$$
\begin{aligned}
M^{\gamma}\left(\Gamma_{1}+Q_{Q_{k}} \Gamma_{2}\right) & =\sum_{(a, b) \in V\left(\Gamma_{1}+Q_{k} \Gamma_{2}\right)} d_{\Gamma_{1}+Q_{k} \Gamma_{2}}^{\gamma}(a, b)=\sum_{(a, b)(c, d) \in E\left(\Gamma_{1}+Q_{k} \Gamma_{2}\right)}\left[d_{\Gamma_{1}+Q_{k} \Gamma_{2}}^{\alpha}(a, b)+d_{\Gamma_{1}+Q_{k} \Gamma_{2}}^{\alpha}(c, d)\right] \\
& =\sum_{a \in V\left(\Gamma_{1}\right)} \sum_{b d \in E\left(\Gamma_{2}\right)}\left[d_{\Gamma_{1}+Q_{k} \Gamma_{2}}^{\alpha}(a, b)+d_{\Gamma_{1}+Q_{k} \Gamma_{2}}^{\alpha}(a, d)\right]+\sum_{b \in V\left(\Gamma_{2}\right)} \sum_{a c \in E\left(Q_{k}\left(\Gamma_{1}\right)\right)}\left[d_{\Gamma_{1}+Q_{k} \Gamma_{2}}^{\alpha}(a, b)+d_{\Gamma_{1}+Q_{k} \Gamma_{2}}^{\alpha}(b, c)\right] .
\end{aligned}
$$

For every vertex $a \in V\left(\Gamma_{1}\right)$ \& edge $b d \in E\left(\Gamma_{2}\right)$, then the 1st term of (25) will be

$$
\begin{aligned}
& \sum_{a \in V\left(\Gamma_{1}\right)} \sum_{b d \in E\left(\Gamma_{2}\right)}\left[d_{\Gamma_{1}+Q_{k} \Gamma_{2}}^{\alpha}(a, b)+d_{\Gamma_{1}+Q_{k} \Gamma_{2}}^{\alpha}(a, d)\right] \\
& =\sum_{a \in V\left(\Gamma_{1}\right)} \sum_{b d \in E\left(\Gamma_{2}\right)}\left[\sum_{i=0}^{\alpha}\left(\begin{array}{c}
\alpha \\
i
\end{array}\right) d_{Q_{k}\left(\Gamma_{1}\right)}^{\alpha-i}(a) d_{\Gamma_{2}}^{i}(b)+\sum_{i=0}^{\alpha}\left(\begin{array}{c}
\alpha \\
i
\end{array}\right) d_{Q_{k}\left(\Gamma_{1}\right)}^{\alpha-i}(a) d_{\Gamma_{2}}^{i}(d)\right] \\
& =\sum_{a \in V\left(\Gamma_{1}\right)} \sum_{b d \in E\left(\Gamma_{2}\right)}\left[\sum_{i=0}^{\alpha}\left(\begin{array}{c}
\alpha \\
i
\end{array}\right) d_{\Gamma_{1}}^{\alpha-i}(a)\left[d_{\Gamma_{2}}^{i}(b)+d_{\Gamma_{2}}^{i}(d)\right]\right] \\
& =\sum_{a \in V\left(\Gamma_{1}\right)}\left[\sum_{i=0}^{\alpha}\left(\begin{array}{c}
\alpha \\
i
\end{array}\right) d_{\Gamma_{1}}^{\alpha-i}(a)\right] \sum_{b d \in E\left(\Gamma_{1}\right)}\left[d_{\Gamma_{2}}^{i}(b)+d_{\Gamma_{2}}^{i}(d)\right] \\
& =\sum_{a \in V\left(\Gamma_{1}\right)}\left[\sum_{i=0}^{\alpha}\left(\begin{array}{c}
\alpha \\
i
\end{array}\right) d_{\Gamma_{1}}^{\alpha-i}(a)\right]\left(M_{\Gamma_{2}}^{i+1}\right) \\
& =\sum_{i=0}^{\alpha}\left(\begin{array}{c}
\alpha \\
i
\end{array}\right)\left(M_{\Gamma_{1}}^{\alpha-i}\right)\left(M_{\Gamma_{2}}^{i+1}\right) .
\end{aligned}
$$

For every vertex $b \in V\left(\Gamma_{2}\right) \& \quad$ edge $a c \in E\left(Q_{k}\left(\Gamma_{1}\right)\right) a, c \in V\left(\Gamma_{1}\right)$, then the 2 nd term of equation (25) will be

$$
\begin{aligned}
& \sum_{b \in V\left(\Gamma_{2}\right)} \sum_{a c \in E\left(Q_{k}\left(\Gamma_{1}\right)\right)}\left[d_{\Gamma_{1}+Q_{k} \Gamma_{2}}^{\alpha}(a, b)+d_{\Gamma_{1}+Q_{k} \Gamma_{2}}^{\alpha}(b, c)\right] \\
& =\sum_{b \in V\left(\Gamma_{2}\right)} \sum_{a c \in E\left(Q_{k}\left(\Gamma_{1}\right)\right) a \in V\left(\Gamma_{1}\right) c \in V\left(Q_{k}\left(\Gamma_{1}\right)\right)-V\left(\Gamma_{1}\right)}\left[d_{\Gamma_{1}+Q_{k} \Gamma_{2}}^{\alpha}(a, b)+d_{\Gamma_{1}+Q_{k} \Gamma_{2}}^{\alpha}(b, c)\right] \\
& \quad \sum_{b \in V\left(\Gamma_{2}\right) a c \in E\left(Q_{k}\left(\Gamma_{1}\right)\right) \backslash \backslash a, c \in V\left(Q_{k}\left(\Gamma_{1}\right)\right)-V\left(\Gamma_{1}\right)}\left[d_{\Gamma_{1}+Q_{k} \Gamma_{2}}^{\alpha}(a, b)+\left[d_{\Gamma_{1}+Q_{k} \Gamma_{2}}^{\alpha}(b, c)\right]\right] .
\end{aligned}
$$


Now $\forall b \in V\left(\Gamma_{2}\right), a c \in E\left(Q_{k}\left(\Gamma_{1}\right)\right)$ if $a \in V\left(\Gamma_{1}\right)$ and $c \in V\left(Q_{k}\left(\Gamma_{1}\right)\right)-V\left(\Gamma_{1}\right)$; the 1 st term of (27) will be

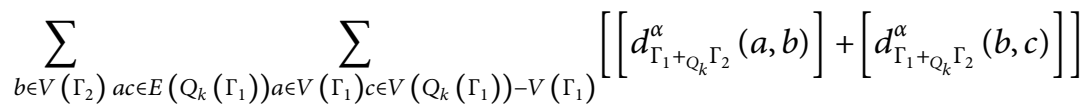

$$
\begin{aligned}
& =\sum_{b \in V\left(\Gamma_{2}\right)} \sum_{a c \in E\left(Q_{k}\left(\Gamma_{1}\right)\right) a \in V\left(\Gamma_{1}\right) c \in V\left(Q_{k}\left(\Gamma_{1}\right)\right)-V\left(\Gamma_{1}\right)}\left[\left[d_{Q_{k}\left(\Gamma_{1}\right)}(a)+d_{\Gamma_{2}}(b)\right]^{\alpha}+\left[d_{Q_{k}\left(\Gamma_{1}\right)}^{\alpha}(c)\right]\right] \\
& =\sum_{b \in V\left(\Gamma_{2}\right)} \sum_{a c \in E\left(Q_{k}\left(\Gamma_{1}\right)\right)} \sum_{a \in V\left(\Gamma_{1}\right)}\left[\sum_{c \in V\left(Q_{k}\left(\Gamma_{1}\right)\right)-V\left(\Gamma_{1}\right)}^{\alpha}\left(\begin{array}{c}
\alpha \\
i
\end{array}\right) d_{Q_{k}\left(\Gamma_{1}\right)}^{\alpha-i}(a) \cdot d_{\Gamma_{2}}^{i}(b)+d_{Q_{k}\left(\Gamma_{1}\right)}^{\alpha}(c)\right] \\
& =\sum_{b \in V\left(\Gamma_{2}\right)} \sum_{a c \in E\left(Q_{k}\left(\Gamma_{1}\right)\right)} \sum_{a \in V\left(\Gamma_{1}\right)}\left[\sum_{c \in V\left(Q_{k}\left(\Gamma_{1}\right)\right)-V\left(\Gamma_{1}\right)}^{\alpha}\left(\begin{array}{c}
\alpha \\
i
\end{array}\right) d_{Q_{k}\left(\Gamma_{1}\right)}^{\alpha-i}(a) \cdot d_{\Gamma_{2}}^{i}(b)\right] \\
& +\sum_{b \in V\left(\Gamma_{2}\right) a c \in E\left(Q_{k}\left(\Gamma_{1}\right)\right)} \sum_{a \in V\left(\Gamma_{1}\right) c \in V\left(Q_{k}\left(\Gamma_{1}\right)\right)-V\left(\Gamma_{1}\right)}\left[d_{Q_{k}\left(\Gamma_{1}\right)}^{\alpha}(c)\right] \\
& =\sum_{i=0}^{\alpha}\left(\begin{array}{c}
\alpha \\
i
\end{array}\right) \sum_{b \in V\left(\Gamma_{2}\right)} d_{\Gamma_{2}}^{i}(b) \sum_{a c \in E\left(Q_{k}\left(\Gamma_{1}\right)\right) a \in V\left(\Gamma_{1}\right) c \in V\left(Q_{k}\left(\Gamma_{1}\right)\right)-V\left(\Gamma_{1}\right)} d_{Q_{k}\left(\Gamma_{1}\right)}^{\alpha-i}(a) \\
& +\sum_{b \in V\left(\Gamma_{2}\right)} \sum_{a c \in E\left(Q_{k}\left(\Gamma_{1}\right)\right) a \in V\left(\Gamma_{1}\right) c \in V\left(Q_{k}\left(\Gamma_{1}\right)\right)-V\left(\Gamma_{1}\right)}\left[d_{Q_{k}\left(\Gamma_{1}\right)}^{\alpha}(c)\right] \\
& =\sum_{i=0}^{\alpha}\left(\begin{array}{c}
\alpha \\
i
\end{array}\right) M_{\Gamma_{2}}^{i} M_{\Gamma_{1}}^{\gamma-i}+n_{\Gamma_{2}} . \sum_{a c \in E\left(Q_{k}\left(\Gamma_{1}\right)\right)} \sum_{a \in V_{\Gamma_{1}} c \in V\left(Q_{k}\left(\Gamma_{1}\right)\right)-V\left(\Gamma_{1}\right)}\left[d_{Q_{k}\left(\Gamma_{1}\right)}^{\alpha}(c)\right] \\
& =\sum_{i=0}^{\alpha}\left(\begin{array}{c}
\alpha \\
i
\end{array}\right) M_{\Gamma_{2}}^{i} M_{\Gamma_{1}}^{\gamma-i}+n_{\Gamma_{2}}\left[2 \sum_{u v \in E\left(\Gamma_{1}\right)}\left(d_{\Gamma_{1}}(u)+d_{\Gamma_{1}}(v)\right)^{\alpha}\right] \\
& =\sum_{i=0}^{\alpha}\left(\begin{array}{c}
\alpha \\
i
\end{array}\right) M_{\Gamma_{2}}^{i} M_{\Gamma_{1}}^{\gamma-i}+2 n_{\Gamma_{2}}\left[\sum_{i=0}^{\alpha}\left(\begin{array}{c}
\alpha \\
i
\end{array}\right) \sum_{u v \in E_{\Gamma_{1}}}\left(d_{\Gamma_{1}}^{\alpha-i}(u) \cdot d_{\Gamma_{1}}^{i}(v)\right] .\right.
\end{aligned}
$$

Now $\forall b \in V\left(\Gamma_{2}\right)$ \& edge $a c \in E\left(Q_{k}\left(\Gamma_{1}\right)\right)$ if the vertex $a, c \in V\left(Q_{k}\left(\Gamma_{1}\right)\right)-V\left(\Gamma_{1}\right)$. Then the 2 nd term of equation
(27) splits into two parts for the vertices $a$ and $c$, then the equation will be 


$$
\begin{aligned}
& =\sum_{b \in V\left(\Gamma_{2}\right)} \sum_{a c \in E\left(Q_{k}\left(\Gamma_{1}\right)\right) a, c \in V\left(Q_{k}\left(\Gamma_{1}\right)\right)-V\left(\Gamma_{1}\right)}\left[d_{\Gamma_{1}+Q_{k} \Gamma_{2}}(a, b)^{\alpha}+d_{\Gamma_{1}+Q_{k} \Gamma_{2}}(b, c)^{\alpha}\right] \\
= & \sum_{b \in V\left(\Gamma_{2}\right)}\left[d_{a c \in E\left(Q_{k}\left(\Gamma_{1}\right)\right) a, c \in V\left(Q_{k}\left(\Gamma_{1}\right)\right)-V\left(\Gamma_{1}\right)}\left[d_{\Gamma_{1}+Q_{k} \Gamma_{2}}(a)^{\alpha}+d_{\Gamma_{1}+Q_{k} \Gamma_{2}}(c)^{\alpha}\right]\right. \\
= & \sum_{b \in V\left(\Gamma_{2}\right)} \sum_{u v \in E\left(\Gamma_{1}\right), v w \in E\left(\Gamma_{1}\right)}\left[d_{\Gamma_{1}}(u)+d_{\Gamma_{1}}(v)\right]^{\alpha}+\left[d_{\Gamma_{1}}(v)+d_{\Gamma_{1}}(w)\right]^{\alpha} \\
= & c_{\Gamma_{2}} \sum_{u v \in E\left(\Gamma_{1}\right), v w \in E\left(\Gamma_{1}\right)}\left[\left[\sum_{i=0}^{\alpha}\left(\begin{array}{c}
\alpha \\
i
\end{array}\right) d_{\Gamma_{1}}^{\alpha-i}(u) \cdot d_{\Gamma_{1}}^{i}(v)\right]+\left[\sum_{i=0}^{\alpha}\left(\begin{array}{c}
\alpha \\
i
\end{array}\right) d_{\Gamma_{1}}^{\alpha-i}(v) \cdot d_{\Gamma_{1}}^{i}(w)\right]\right] \\
\sum_{b s V\left(\Gamma_{2}\right) a c \varepsilon E\left(Q_{k}\left(\Gamma_{1}\right)\right) a c \varepsilon V\left(Q_{k}\left(\Gamma_{1}\right)\right)-V\left(\Gamma_{1}\right)}\left[d_{Q_{k}\left(\Gamma_{1}\right)}^{\alpha}(a)+d_{Q_{k}\left(\Gamma_{1}\right)}^{\alpha}(c)\right] & =2(k-1) \sum_{b s V\left(\Gamma_{2}\right)} \sum_{u v \varepsilon E\left(\Gamma_{1}\right)}\left[d_{\Gamma_{1}}^{\alpha}(u)+d_{\Gamma_{1}}^{\alpha}(v)\right] \\
& =2(k-1) n_{\Gamma_{2}} \sum_{u v \varepsilon E\left(\Gamma_{1}\right)}\left[d_{\Gamma_{1}}^{\alpha}(u)+d_{\Gamma_{1}}^{\alpha}(v)\right] .
\end{aligned}
$$

Using (26), (28), (29), and (30) in (25), we get the required result:

$$
\begin{aligned}
= & \sum_{i=0}^{\alpha}\left(\begin{array}{c}
\alpha \\
i
\end{array}\right)\left(M _ { \Gamma _ { 1 } } ^ { \alpha - i } \left(M_{\Gamma_{2}}^{i+1}+\sum_{i=0}^{\alpha}\left(\begin{array}{c}
\alpha \\
i
\end{array}\right) M_{\Gamma_{2}}^{i} M_{\Gamma_{1}}^{\alpha}\right.\right. \\
& +2 n_{\Gamma_{2}} \sum_{i=0}^{\alpha}\left(\begin{array}{c}
\alpha \\
i
\end{array}\right) \sum_{u v \in E^{\prime}\left(\Gamma_{1}\right)}\left(d_{\Gamma_{1}}^{\alpha-i}(u) \cdot d_{\Gamma_{1}}^{i}(v)\right] \\
& +n_{\Gamma_{2}} \sum_{u v \in E^{\prime}\left(\Gamma_{1}\right), v w \in E^{\prime}\left(\Gamma_{1}\right)}\left[\sum_{i=0}^{\alpha}\left[\left(\begin{array}{c}
\alpha \\
i
\end{array}\right) d_{\Gamma_{1}}^{\alpha-i}(u) \cdot d_{\Gamma_{1}}^{i}(v)\right]\right]+\left[\sum_{i=0}^{\alpha}\left(\begin{array}{c}
\alpha \\
i
\end{array}\right) d_{\Gamma_{1}}^{\alpha-i}(v) \cdot d_{\Gamma_{1}}^{i}(w)\right]+2\left(k-1 n_{\Gamma_{2}} \sum_{u v E E\left(\Gamma_{1}\right)}\left[d_{\Gamma_{1}}^{\alpha}(u)+d_{\Gamma_{1}}^{\alpha}(v)\right]\right. \\
= & \sum_{i=0}^{\alpha}\left(\begin{array}{c}
\alpha \\
i
\end{array}\right)\left(M_{\Gamma_{1}}^{2 \gamma-2-i}\left(M_{\Gamma_{2}}^{2 i+1}\right)+2 n_{\Gamma_{2}} \sum_{i=0}^{\alpha}\left(\begin{array}{c}
\alpha \\
i
\end{array}\right) \sum_{u v \in E\left(\Gamma_{1}\right)}\left(d_{\Gamma_{1}}^{\alpha-i}(u) \cdot d_{\Gamma_{1}}^{i}(v)\right]+n_{\Gamma_{2}} \sum_{u v \in E\left(\Gamma_{1}\right), v w \in E\left(\Gamma_{1}\right)}\left[\left[\sum_{i=0}^{\alpha}\left(\begin{array}{c}
\alpha \\
i
\end{array}\right) d_{\Gamma_{1}}^{\alpha-i}(u) \cdot d_{\Gamma_{1}}^{i}(v)\right]\right.\right. \\
& \left.+\left[\sum_{i=0}^{\alpha}\left(\begin{array}{c}
\alpha \\
i
\end{array}\right) d_{\Gamma_{1}}^{\alpha-i}(v) \cdot d_{\Gamma_{1}}^{i}(w)\right]\right]+2(k-1) n_{\Gamma_{2}} \sum_{u v \varepsilon E\left(\Gamma_{1}\right)}\left[d_{\Gamma_{1}}^{\alpha}(u)+d_{\Gamma_{1}}^{\alpha}(v)\right] .
\end{aligned}
$$

Theorem 4. Let $\Gamma_{1}$ and $\Gamma_{2}$ be two simple graphs. The FGZ index of the generalized T-sum graph $\Gamma_{1}+_{T_{k}} \Gamma_{2}$ is

$$
\begin{aligned}
M^{\gamma}\left(\Gamma_{1}+T_{T_{k}} \Gamma_{2}\right)= & \sum_{i=0}^{\alpha}\left(\begin{array}{c}
\alpha \\
i
\end{array}\right)(2)^{\alpha-i} M_{\Gamma_{1}}^{\alpha-i} M_{\Gamma_{2}}^{i+1}+\sum_{i=0}^{\alpha}\left(\begin{array}{c}
\alpha \\
i
\end{array}\right) M_{\Gamma_{2}}^{i}(2)^{(\alpha-i)} M_{\Gamma_{1}}^{\gamma-i}+\sum_{i=0}^{\alpha}\left(\begin{array}{c}
\alpha \\
i
\end{array}\right)(2)^{\alpha-i} M_{\Gamma_{2}}^{i} M_{\Gamma_{1}}^{\gamma-i} \\
& +2 n_{\Gamma_{2}} \sum_{i=0}^{\alpha}\left(\begin{array}{c}
\alpha \\
i
\end{array}\right) \sum_{u v \in E\left(\Gamma_{1}\right)}\left(d_{\Gamma_{1}}^{\alpha-i}(u) \cdot d_{\Gamma_{1}}^{i}(v)\right]+n_{\Gamma_{2}} \\
& \cdot \sum_{u v \in E\left(\Gamma_{1}\right), v w \in E\left(\Gamma_{1}\right)}\left[\left[\sum_{i=0}^{\alpha}\left(\begin{array}{c}
\alpha \\
i
\end{array}\right)\left(d_{\Gamma_{1}}^{\alpha-i}(u) \cdot d_{\Gamma_{1}}^{i}(v)\right]+\left[\sum_{i=0}^{\alpha}\left(\begin{array}{c}
\alpha \\
i
\end{array}\right) d_{\Gamma_{1}}^{\alpha-i}(v)+d_{\Gamma_{1}}^{i}(w)\right]\right]\right] \\
& +2(k-1) n_{\Gamma_{2}} \sum_{u v \in E\left(\Gamma_{1}\right)}\left[d_{\Gamma_{1}}^{\alpha}(u)+d_{\Gamma_{1}}^{\alpha}(v)\right],
\end{aligned}
$$


where $\gamma \in N^{+}-\{0,1\}$ and $\alpha=\gamma-1$.

Proof. Since we have $d_{\Gamma_{1}+T_{k} \Gamma_{2}}(a, b)=d_{\Gamma_{1}+R_{R} \Gamma_{2}}(a, b)$ for every vertex $a \in V\left(\Gamma_{1}\right) \quad$ and $\quad b \in V\left(\Gamma_{2}\right), \quad$ also $d_{\Gamma_{1}+T_{k} \Gamma_{2}}(a, b)=d_{\Gamma_{1}+Q_{k} \Gamma_{2}}(a, b) \quad$ for $\quad$ every vertex $a \in V\left(T_{k}\left(\Gamma_{1}\right)\right)-V\left(\Gamma_{1}\right)$ and $b \in V\left(\Gamma_{2}\right)$, the result follows by the proof of Theorems 2 and 3.
Theorem 5. Assume that $\Gamma_{1}$ and $\Gamma_{2}$ are two simple graphs and $\alpha=\gamma-1$, where $\gamma \in \mathfrak{R}-\left\{0, N^{+}\right\}$and $\mathfrak{R}$ is a set of real number. Then, the FGZ index of generalized F-sum graphs $\left(\Gamma_{1}+{ }_{S_{k}} \Gamma_{2}, \Gamma_{1}+{ }_{R_{k}} \Gamma_{2}, \Gamma_{1}+{ }_{Q_{k}} \Gamma_{2}\right.$, and $\left.\Gamma_{1}+_{T_{k}} \Gamma_{2}\right)$ are

(i) $M^{\gamma}\left(\Gamma_{1}+_{S_{k}} \Gamma_{2}\right)=\sum_{i=0}^{\infty}\left(\begin{array}{c}\alpha \\ i\end{array}\right)\left(M_{\Gamma_{1}}^{i}\right)\left(M_{\Gamma_{2}}^{\alpha-i+1}\right)+n_{\Gamma_{2}} M_{S_{k}\left(\Gamma_{1}\right)}^{i}+\sum_{i=1}^{\infty}\left(\begin{array}{l}\alpha \\ i\end{array}\right) M_{\Gamma_{2}}^{\alpha-1} M_{\Gamma_{1}}^{i+1}+2^{\gamma+1}(k-1) n_{\Gamma_{2}} e_{\Gamma_{1}}$

(ii) $M^{\gamma}\left(\Gamma_{1}+R_{k} \Gamma_{2}\right)=\sum_{i=0}^{\infty}\left(\begin{array}{c}\alpha \\ i\end{array}\right) 2^{i} M_{\Gamma_{1}}^{i} M_{\Gamma_{2}}^{\alpha-i+1}+2\left[\sum_{i=0}^{\infty}\left[\left(\begin{array}{c}\alpha \\ i\end{array}\right) 2^{i} M_{\Gamma_{1}}^{i+1} M_{\Gamma_{2}}^{\alpha-i}\right]\right]+2^{\gamma} e_{\Gamma_{1}} n_{\Gamma_{2}}+2^{\gamma+1}(k-1) n_{\Gamma_{2}} e_{\Gamma_{1}}$

(iii) $M_{\Gamma_{1}+Q_{k} \Gamma_{2}}^{\gamma}=\sum_{i=0}^{\infty}\left(\begin{array}{c}\alpha \\ i\end{array}\right)\left(M_{\Gamma_{1}}^{i}\right)\left(M_{\Gamma_{2}}^{\alpha-i+1}\right)+\sum_{i=0}^{\infty}\left(\begin{array}{c}\alpha \\ i\end{array}\right)\left(M_{\Gamma_{1}}^{i+1}\right)\left(M_{\Gamma_{2}}^{\alpha-i}\right)+2 n_{\Gamma_{2}}\left[\sum_{i=0}^{\infty}\left(\begin{array}{c}\alpha \\ i\end{array}\right)\left(d_{\Gamma_{1}}^{i}(u) \cdot d_{\Gamma_{1}}^{\alpha-i}(v)\right]\right.$

$+n_{\Gamma_{2}} \sum_{u v \in E\left(\Gamma_{1}\right), v w \in E\left(\Gamma_{1}\right)}\left[\left[\sum_{i=0}^{\infty}\left(\begin{array}{c}\alpha \\ i\end{array}\right) d_{\Gamma_{1}}^{i}(u) \cdot d_{\Gamma_{1}}^{\alpha-i}(v)\right]+\left[\sum_{i=0}^{\infty}\left[\left(\begin{array}{c}\alpha \\ i\end{array}\right) d_{\Gamma_{1}}^{i}(v) \cdot d_{\Gamma_{1}}^{\alpha-i}(w)\right]\right]\right.$

$+2(k-1) n_{\Gamma_{2}} \sum_{u v \varepsilon E\left(\Gamma_{1}\right)}\left[d_{\Gamma_{1}}^{\gamma}(u)+d_{\Gamma_{1}}^{\gamma}(v)\right]$

(iv) $M^{\gamma}\left(\Gamma_{1}+T_{k} \Gamma_{2}\right)=\sum_{i=0}^{\infty}\left(\begin{array}{c}\alpha \\ i\end{array}\right) 2^{i} M_{\Gamma_{1}}^{i} M_{\Gamma_{2}}^{\alpha-i+1}+\left[\sum_{i=0}^{\infty}\left(\begin{array}{c}\alpha \\ i\end{array}\right) 2^{i} M_{\Gamma_{1}}^{i+1} M_{\Gamma_{2}}^{\alpha-i}\right]$

$+\sum_{i=0}^{\infty}\left(\begin{array}{c}\alpha \\ i\end{array}\right)\left(M_{\Gamma_{1}}^{i+1}\right)\left(M_{\Gamma_{2}}^{\alpha-i}\right)+2 n_{\Gamma_{2}} \sum_{i=0}^{\infty}\left(\begin{array}{c}\alpha \\ i\end{array}\right) \sum_{u v \in E\left(\Gamma_{1}\right)}\left(d_{\Gamma_{1}}^{i}(u) \cdot d_{\Gamma_{1}}^{\alpha-i}(v)\right.$

$+n_{\Gamma_{2}} \sum_{u v \in E\left(\Gamma_{1}\right), v w \in E\left(\Gamma_{1}\right)}\left[\left[\sum_{i=0}^{\infty}\left(\begin{array}{c}\alpha \\ i\end{array}\right) d_{\Gamma_{1}}^{i}(u) \cdot d_{\Gamma_{1}}^{\alpha-i}(v)\right]+\left[\sum_{i=0}^{\infty}\left(\begin{array}{c}\alpha \\ i\end{array}\right) d_{\Gamma_{1}}^{i}(v) \cdot d_{\Gamma_{1}}^{\alpha-i}(w)\right]\right]$

$+2(k-1) n_{\Gamma_{2}} \sum_{u v \varepsilon E\left(\Gamma_{1}\right)}\left[d_{\Gamma_{1}}^{\gamma}(u)+d_{\Gamma_{1}}^{\gamma}(v)\right]$. 
Proof. The above proof is similar as of Theorems 1-4.

Let $\Gamma_{1}$ be a negative integer, so from Theorem 5, Corollary 1 is obtained.
Corollary 1. Assume that $\Gamma_{1} \& \Gamma_{2}$ are two simple graphs and $\alpha=\gamma-1$, where $\gamma$ is a negative real number. The FGZ index of the generalized F-sums graphs $\left(\Gamma_{1}+{ }_{S_{k}} \Gamma_{2}, \Gamma_{1}+{ }_{R_{k}} \Gamma_{2}, \Gamma_{1}+{ }_{Q_{k}} \Gamma_{2}\right.$, and $\Gamma_{1}+_{T_{k}} \Gamma_{2}$ ) are

$$
\begin{aligned}
(i) M^{\gamma}\left(\Gamma_{1}+{ }_{S_{k}} \Gamma_{2}\right)= & \sum_{i=0}^{\infty}(-1)^{i}\left(\begin{array}{c}
\alpha+i-1 \\
i
\end{array}\right)\left(M_{\Gamma_{1}}^{i}\right)\left(M_{\Gamma_{2}}^{\alpha-i+1}\right)+n_{\Gamma_{2}} M_{S_{k}\left(\Gamma_{1}\right)}^{i}+\sum_{i=1}^{\infty}(-1)^{i}\left(\begin{array}{c}
\alpha+i-1 \\
i
\end{array}\right) M_{\Gamma_{2}}^{\alpha-1} M_{\Gamma_{1}}^{i+1}+2^{\gamma+1}(k-1) n_{\Gamma_{2}} e_{\Gamma_{1}} \\
(i i) M^{\gamma}\left(\Gamma_{1}+{ }_{R_{k}} \Gamma_{2}\right)= & \sum_{i=0}^{\infty}(-1)^{i}\left(\begin{array}{c}
\alpha+i-1 \\
i
\end{array}\right) 2^{i} M_{\Gamma_{1}}^{i} M_{\Gamma_{2}}^{\alpha-i+1}+2\left[\sum_{i=0}^{\infty}(-1)^{i}\left(\begin{array}{c}
\alpha+i-1 \\
i
\end{array}\right) 2^{i} M_{\Gamma_{1}}^{i+1} M_{\Gamma_{2}}^{\alpha-i}\right]+2^{\gamma} e_{\Gamma_{1}} n_{\Gamma_{2}}+2^{\gamma+1}(k-1) n_{\Gamma_{2}} e_{\Gamma_{1}} \\
(i i i) M_{\Gamma_{1}+Q_{k} \Gamma_{2}}^{\gamma}= & \sum_{i=0}^{\infty}(-1)^{i}\left(\begin{array}{c}
\alpha+i-1 \\
i
\end{array}\right)\left(M_{\Gamma_{1}}^{i}\right)\left(M_{\Gamma_{2}}^{\alpha-i+1}\right)+\sum_{i=0}^{\infty}(-1)^{i}\left(\begin{array}{c}
\alpha+i-1 \\
i
\end{array}\right)\left(M_{\Gamma_{1}}^{i+1}\right)\left(M_{\Gamma_{2}}^{\alpha-i}\right) \\
& +2 n_{\Gamma_{2}} \sum_{i=0}^{\infty}(-1)^{i}\left(\begin{array}{c}
\alpha+i-1 \\
i
\end{array}\right) \sum_{u v \in E\left(\Gamma_{1}\right)}\left(d_{\Gamma_{1}}^{i}(u) \cdot d_{\Gamma_{1}}^{\alpha-i}(v)+n_{\Gamma_{2}} \sum_{u v \in E\left(\Gamma_{1}\right), v w \in E\left(\Gamma_{1}\right)}\left[\left[\sum_{i=0}^{\infty}(-1)^{i}\left(\begin{array}{c}
\alpha+i-1 \\
i
\end{array}\right) d_{\Gamma_{1}}^{i}(u) \cdot d_{\Gamma_{1}}^{\alpha-i}(v)\right]\right.\right. \\
& \left.+\left[\sum_{i=0}^{\infty}(-1)^{i}\left(\begin{array}{c}
\alpha+i-1 \\
i
\end{array}\right) d_{\Gamma_{1}}^{i}(v) \cdot d_{\Gamma_{1}}^{\alpha-i}(w)\right]\right]+2(k-1) n_{\Gamma_{2}} \sum_{u v \varepsilon E\left(\Gamma_{1}\right)}\left[d_{\Gamma_{1}}^{\gamma}(u)+d_{\Gamma_{1}}^{\gamma}(v)\right] \\
(i v) M^{\gamma}\left(\Gamma_{1}+T_{k} \Gamma_{2}\right)= & \sum_{i=0}^{\infty}(-1)^{i}\left(\begin{array}{c}
\alpha+i-1 \\
i
\end{array}\right) 2^{i} M_{\Gamma_{1}}^{i} M_{\Gamma_{2}}^{\alpha-i+1}+\left[\sum_{i=0}^{\infty}(-1)^{i}\left(\begin{array}{c}
\alpha+i-1 \\
i
\end{array}\right) 2^{i} M_{\Gamma_{1}}^{i+1} M_{\Gamma_{2}}^{\alpha-i}\right] \\
& +\sum_{i=0}^{\infty}(-1)^{i}\left(\begin{array}{c}
\alpha+i-1 \\
i
\end{array}\right)\left(M_{\Gamma_{1}}^{i+1}\right)\left(M_{\Gamma_{2}}^{\alpha-i}\right)+2 n_{\Gamma_{2}} \sum_{i=0}^{\infty}(-1)^{i}\left(\begin{array}{c}
\alpha+i-1 \\
i
\end{array}\right) \sum_{u v \in E\left(\Gamma_{1}\right)}\left(d_{\Gamma_{1}}^{i}(u) \cdot d_{\Gamma_{1}}^{\alpha-i}(v)\right. \\
& +n_{\Gamma_{2}} \sum_{u v \in E\left(\Gamma_{1}\right), v w \in E\left(\Gamma_{1}\right)}\left[\left[\sum_{i=0}^{\infty}(-1)^{i}\left(\begin{array}{c}
\alpha+i-1 \\
i
\end{array}\right) d_{\Gamma_{1}}^{i}(u) \cdot d_{\Gamma_{1}}^{\alpha-i}(v)\right]+\left[\sum_{i=0}^{\infty}(-1)^{i}\left(\begin{array}{c}
\alpha+i-1 \\
i
\end{array}\right) d_{\Gamma_{1}}^{i}(v) \cdot d_{\Gamma_{1}}^{\alpha-i}(w)\right]\right] \\
& +2(k-1) n_{\Gamma_{2}} \sum_{u v \varepsilon E\left(\Gamma_{1}\right)}\left[d_{\Gamma_{1}}^{\gamma}(u)+d_{\Gamma_{1}}^{\gamma}(v)\right] .
\end{aligned}
$$

\section{Applications}

Now, we present some examples as applications of the obtained results Theorems 1-4. Also the numerical comparisons are represented in Tables 1-4, and the graphical representations are depicted in Figures 4-7.
Example 1. Let $P_{m}$ and $P_{n}$ be two simple graphs with $m \geq 2$ and $n \geq 2$. Then, we have

$$
\text { 1. } \begin{aligned}
M^{\gamma}\left(P_{m+S_{k}} P_{n}\right)= & \sum_{t=0}^{\gamma} C_{T}^{\gamma-1}\left[2^{\gamma-1-t}(m-2)+2\right]\left[2^{t+1}(n-2)+2\right]+\sum_{t=0}^{\gamma} C_{T}^{\gamma-1}\left[2^{\gamma-t}(m-2)+2\right]\left[2^{t}(n-2)+2\right] \\
& +n\left(2^{\gamma}(2 m-3)+2\right)+4(k-1) n(m-1) .
\end{aligned}
$$

From Figure 4, it is clear that the behavior of FGZ index of the generalized $S$-sum graph $\Gamma_{1}+{ }_{S_{k}} \Gamma_{2}$ at $t=2$ is more better than $t=0$ and $t=1$ :

$$
\text { 2. } \begin{aligned}
M^{\gamma}\left(P_{m+R_{k}} P_{n}\right)= & \sum_{t=0}^{\gamma} C_{T}^{\gamma-1} 2^{\gamma-1-t}\left[2^{\gamma-1-t}(m-2)+2\right] \cdot\left[2^{t+1}(n-2)+2\right]+2 \sum_{t=0}^{\gamma} C_{T}^{\gamma-1} 2^{\gamma-1-t}\left[2^{\gamma-t}(m-2)+2\right]\left[2^{t}(n-2)+2\right] \\
& +2(m-1) n+4(k-1) n(m-1) .
\end{aligned}
$$


TABle 1: Numerical comparison for $M^{\gamma}\left(P_{m+S_{k}} P_{n}\right)$.

\begin{tabular}{lccc}
\hline$[\mathrm{m}, n, k]$ & $T=0$ & $T=1$ & $T=2$ \\
\hline$[1,1,1]$ & -4 & -4 & -13 \\
{$[2,2,2]$} & 28 & 28 & 28 \\
{$[3,3,3]$} & 124 & 124 & 133 \\
{$[4,4,4]$} & 308 & 308 & 326 \\
{$[5,5,5]$} & 604 & 604 & 631 \\
{$[6,6,6]$} & 1036 & 1036 & 1072 \\
{$[7,7,7]$} & 1628 & 1628 & 1673 \\
{$[8,8,8]$} & 2404 & 2404 & 2458 \\
{$[9,9,9]$} & 3388 & 3388 & 3451 \\
{$[10,10,10]$} & 4604 & 4604 & 4676 \\
\hline
\end{tabular}

TABle 2: Numerical comparison for $M^{\gamma}\left(P_{m+R_{k}} P_{n}\right)$.

\begin{tabular}{lccc}
\hline$[\mathrm{m}, n, \mathrm{k}]$ & $T=0$ & $T=1$ & $T=2$ \\
\hline$[1,1,1]$ & -8 & -2 & -6.5000 \\
{$[2,2,2]$} & 36 & 24 & 18 \\
{$[3,3,3]$} & 164 & 110 & 90.5000 \\
{$[4,4,4]$} & 400 & 280 & 235 \\
{$[5,5,5]$} & 768 & 558 & 475.000 \\
{$[6,6,6]$} & 1292 & 968 & 836 \\
{$[7,7,7]$} & 1996 & 1534 & 1340.5 \\
{$[8,8,8]$} & 2904 & 2280 & 2013 \\
{$[9,9,9]$} & 4040 & 3230 & 2877.5 \\
{$[10,10,10]$} & 5428 & 4408 & 3958 \\
\hline
\end{tabular}

TABLE 3: Numerical comparison for $M^{\gamma}\left(P_{m+Q_{k}} P_{n}\right)$.

\begin{tabular}{lccc}
\hline$[\mathrm{m}, n, k]$ & $T=0$ & $T=1$ & $T=2$ \\
\hline$[1,1,1]$ & 3 & 3 & -1.5000 \\
{$[2,2,2]$} & 38 & 38 & 47 \\
{$[3,3,3]$} & 157 & 157 & 179.5000 \\
{$[4,4,4]$} & 408 & 408 & 444 \\
{$[5,5,5]$} & 839 & 839 & 888.5000 \\
{$[6,6,6]$} & 1498 & 1498 & 1561 \\
{$[7,7,7]$} & 2433 & 2433 & 2509.5 \\
{$[8,8,8]$} & 3692 & 3692 & 3782 \\
{$[9,9,9]$} & 5323 & 5323 & 5426.5 \\
{$[10,10,10]$} & 7374 & 7374 & 7491 \\
\hline
\end{tabular}

TABle 4: Numerical comparison for $M^{\gamma}\left(P_{m+T_{k}} P_{n}\right)$.

\begin{tabular}{lccc}
\hline$[m, n, k]$ & $T=0$ & $T=1$ & $T=2$ \\
\hline$[1,1,1]$ & -3 & 3 & 3 \\
{$[2,2,2]$} & 54 & 42 & 45 \\
{$[3,3,3]$} & 227 & 173 & 167 \\
{$[4,4,4]$} & 564 & 444 & 417 \\
{$[5,5,5]$} & 1113 & 903 & 843 \\
{$[6,6,6]$} & 1922 & 1598 & 1493 \\
{$[7,7,7]$} & 3039 & 2577 & 2415 \\
{$[8,8,8]$} & 4512 & 3888 & 3657 \\
{$[9,9,9]$} & 6389 & 5579 & 5267 \\
{$[10,10,10]$} & 8718 & 7698 & 7293 \\
\hline
\end{tabular}

From Figure 5, it is clear that the behavior of FGZ index of the generalized $R$-sum graph $\Gamma_{1}+{ }_{R_{k}} \Gamma_{2}$ at $t=0$ is more better than $t=1$ and $t=2$ :

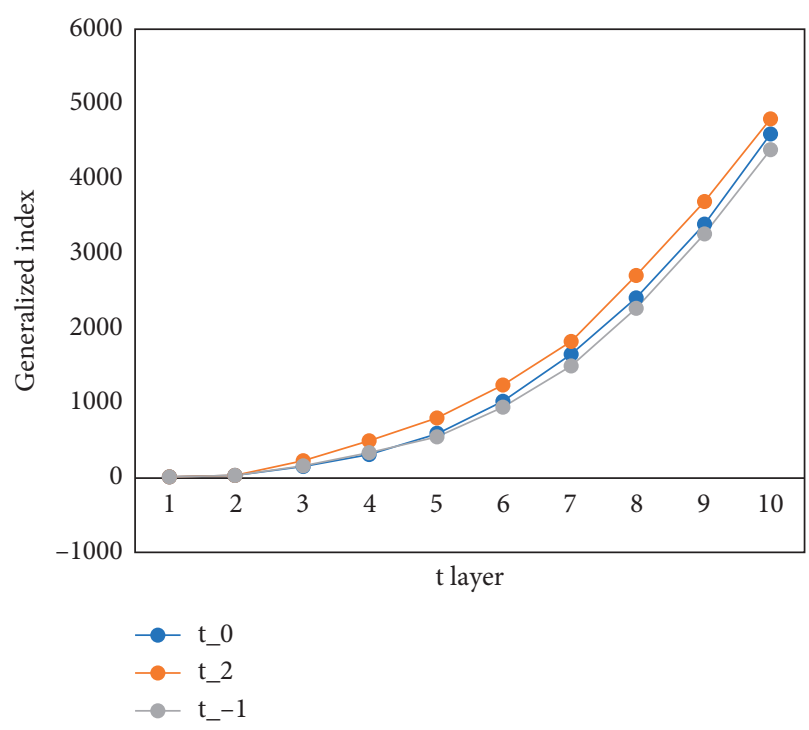

Figure 4: Numerical behavior of $M^{\gamma}\left(P_{m+S_{k}} P_{n}\right)$ using Table 1.

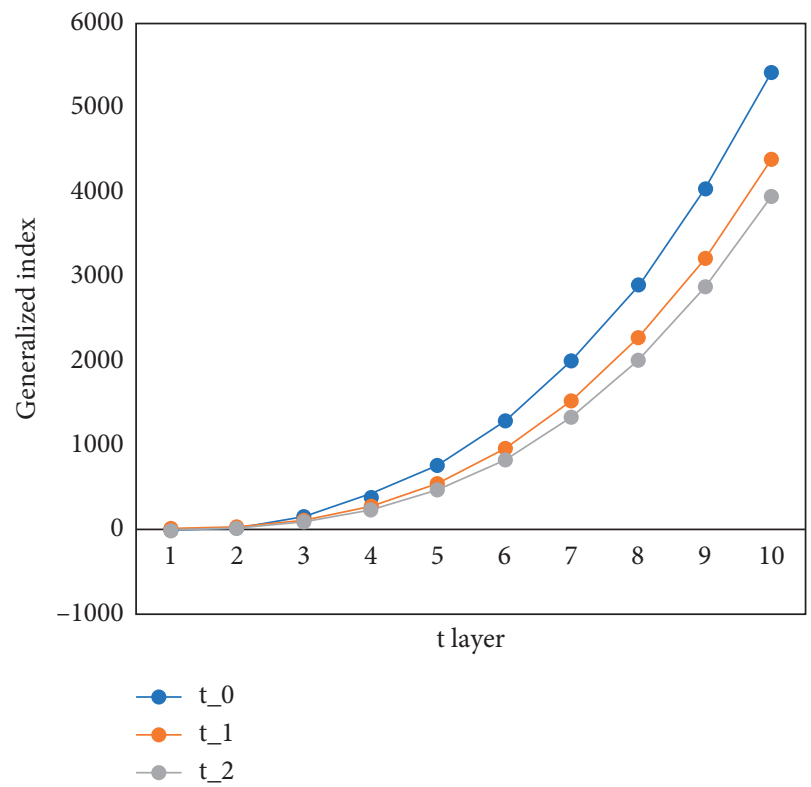

Figure 5: Numerical behavior of $M^{\gamma}\left(P_{m+R_{k}} P_{n}\right)$ using Table 2.

$$
\text { 3. } \begin{aligned}
M^{\gamma}\left(P_{m+Q_{k}} P_{n}\right)= & \sum_{t=0}^{\gamma} C_{T}^{\gamma-1}\left[2^{\gamma-1-t}(m-2)+2\right]\left[2^{t+1}(n-2)+2\right] \\
& +\sum_{t=0}^{\gamma} C_{T}^{\gamma-1}\left[2^{\gamma-t}(m-2)+2\right]\left[2^{t}(n-2)+2\right] \\
& +2 n \sum_{t=0}^{\gamma} C_{T}^{\gamma-1}\left[2^{\gamma-1-t}+(m-2) 2^{\gamma-1}+2^{t}\right] \\
& +n \sum_{t=0}^{\gamma} C_{T}^{\gamma-1}\left[2^{t}+2(m-1) 2^{\gamma-1}+2^{\gamma-1-t}\right] \\
& \cdot 2(k-1) n\left[2^{\alpha+1}(m-2)+2\right] .
\end{aligned}
$$




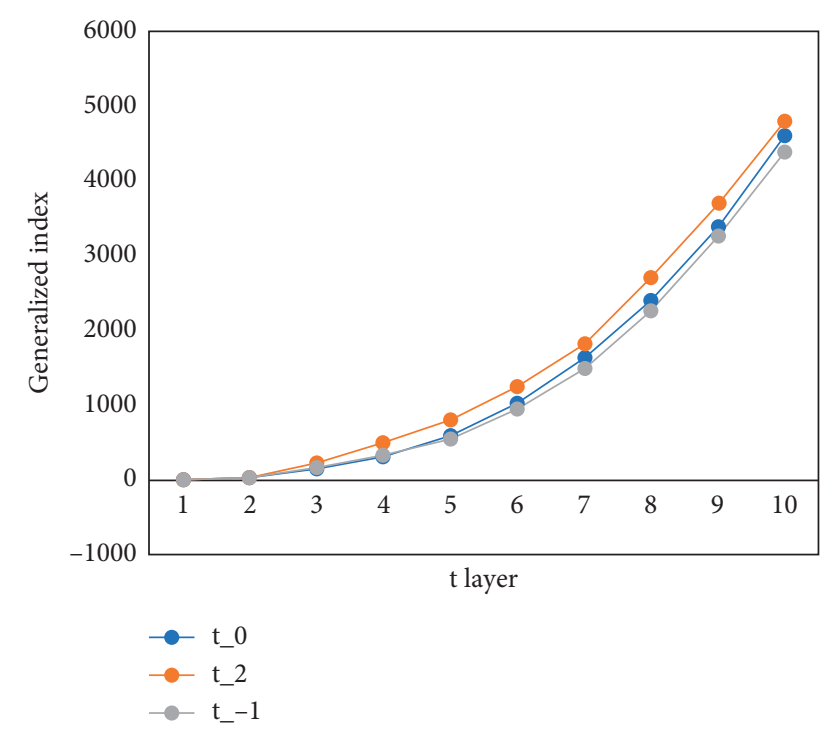

Figure 6: Numerical behavior of $M^{\gamma}\left(P_{m+Q_{k}} P_{n}\right)$ using Table 3.

From Figure 6, it is clear that the behavior of FGZ index of the generalized $Q$-sum graph $\Gamma_{1}{ }^{+}{ }_{Q_{k}} \Gamma_{2}$ at $t=2$ is more better than $t=0$ and $t=1$ :

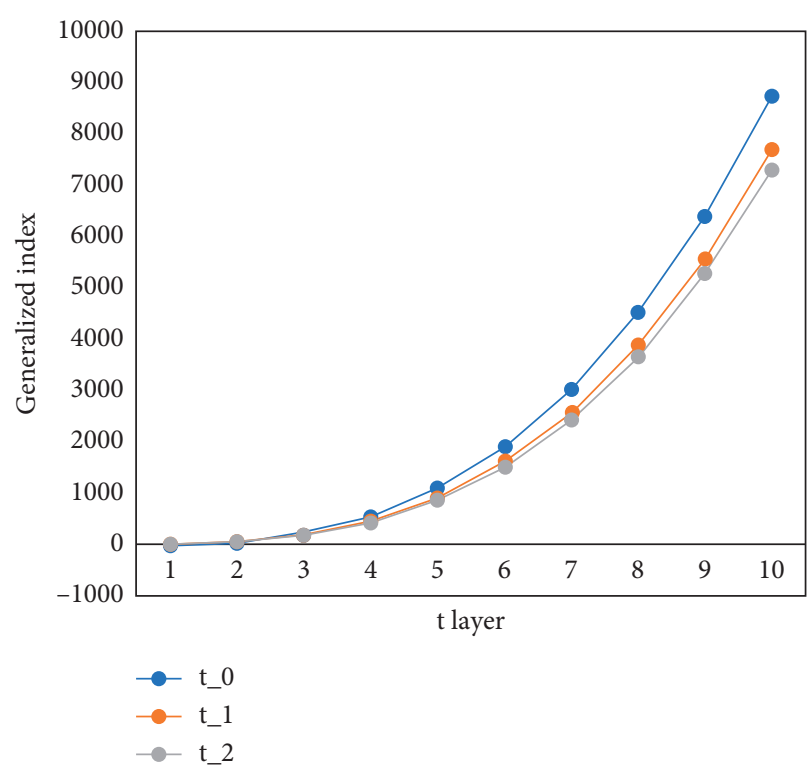

FIgURE 7: Numerical behavior of $M^{\gamma}\left(P_{m+T_{k}} P_{n}\right)$.

$$
\begin{aligned}
\text { 4. } M^{\gamma}\left(P_{m+T_{k}} P_{n}\right)= & \sum_{t=0}^{\gamma} C_{T}^{\gamma-1} 2^{\gamma-1-t}\left[2^{\gamma-1-t}(m-2)+2\right]\left[2^{t+1}(n-2)+2\right]+2 \sum_{t=0}^{\gamma} C_{T}^{\gamma-1} 2^{\gamma-1-t}\left[2^{\gamma-t}(m-2)+2\right]\left[2^{t}(n-2)+2\right] \\
& +2 n \sum_{t=0}^{\gamma} C_{T}^{\gamma-1}\left[2^{\gamma-1-t}+(m-2) 2^{\gamma-1}+2^{t}\right]+n \sum_{t=0}^{\gamma} C_{T}^{\gamma-1}\left[2^{t}+2(m-1) 2^{\gamma-1}+2^{\gamma-1-t}\right] 2(k-1) n\left[2^{\alpha+1}(m-2)+2\right] .
\end{aligned}
$$

From Figure 7, it is clear that the behavior of FGZ index of the generalized $T$-sum graph $\Gamma_{1}+_{T_{k}} \Gamma_{2}$ at $t=0$ is more better than $t=1$ and $t=2$.

\section{Conclusions}

Now, we close our discussion with the following remarks:

(i) For positive integer $k$ and two graphs $\Gamma_{1} \& \Gamma_{2}$, we have computed FGZ index of the generalized $F$-sums graphs $\Gamma_{1+F_{k}} \Gamma_{2}$, where generalized $F$-sums graphs are obtained by the different operations of subdivision and Cartesian product on $\Gamma_{1} \& \Gamma_{2}$.

(ii) The obtained results are also verified and illustrated for the particular classes of graphs.

(iii) The behavior of FGZ index is also analyzed with the help of numerical and graphical presentations.

(iv) However, the problem is still open to compute the different topological indices (degree and distance based) for the generalized F-sum graphs.

\section{Data Availability}

All the data are included within this paper. However, the reader may contact the corresponding author for more details of the data.

\section{Conflicts of Interest}

The authors have no conflicts of interest.

\section{Acknowledgments}

The authors are also thankful to Dr. Muhammad Kamran Siddiqui who helped in the graphical analysis. The University of Hail, Saudi Arabia, partially supported the study.

\section{References}

[1] H. Gonzalez-Diaz, S. Vilar, L. Santana, and E. Uriarte, "Medicinal chemistry and bioinformatics-current trends in drugs discovery with networks topological indices," Current Topics in Medicinal Chemistry, vol. 7, pp. 1025-1039, 2007.

[2] R. Gozalbes, J. Doucet, and F. Derouin, "Application of topological descriptors in QSAR and drug design: history and 
new trends," Current Drug Target-Infectious Disorders, vol. 2, no. 1, pp. 93-102, 2002.

[3] G. Rücker and C. Rücker, "On topological indices, boiling points, and cycloalkanes," Journal of Chemical Information and Computer Sciences, vol. 39, no. 5, pp. 788-802, 1999.

[4] M. Randic, "On characterization of molecular branching," Journal of the American Chemical Society, vol. 97, pp. 66096615, 1975.

[5] A. R. Matamala and E. Estrada, "Generalised topological indices: optimisation methodology and physico-chemical interpretation," Chemical Physics Letters, vol. 410, no. 4-6, pp. 343-347, 2005.

[6] W. Yan, B.-Y. Yang, and Y.-N. Yeh, "The behavior of Wiener indices and polynomials of graphs under five graph decorations," Applied Mathematics Letters, vol. 20, no. 3, pp. 290-295, 2007.

[7] H. Gonzglez-Diaz, S. Vilar, L. Santana, and E. Uriarte, "Medicinal chemistry and bioinformatics-current trends in drugs discovery with networks topological indices," Current Topics in Medicinal Chemistry, vol. 7, no. 10, pp. 1015-1029, 2007.

[8] L. H. Hall and L. B. Kier, Molecular Connectivity in Chemistry and Drug Research, Academic Press, Boston, MA, USA, 1976.

[9] M. V. Diudea, QSPR/QSAR Studies by Molecular Descriptors, NOVA, New York, NY, USA, 2001.

[10] J. Devillers and A. T. Balaban, Topological Indices and Related Descriptors in QSAR and QSPR, Gordon \& Breach, Amsterdam, Netherlands, 1999.

[11] R. Todeschini, V. Consonni, R. Mannhold, H. Kubinyi, and H. Timmerman, Handbook of Molecular Descriptors, WileyVCH, Weinheim, Germany, 2002.

[12] I. Gutman and O. Polansky, Mathematical Concepts in Organic Chemistry, Springer-Verlag, Berlin, Germany, 1986.

[13] I. Gutman, "Degree-based topological indices," Croatica Chemica Acta, vol. 86, no. 4, pp. 351-361, 2013.

[14] H. Wiener, "Structural determination of paraffin boiling points," Journal of the American Chemical Society, vol. 69, no. 1, pp. 17-20, 1947.

[15] N. Trinajstić and I. Gutman, "Graph theory and molecular orbitals. Total $\varphi$-electron energy of alternant hydrocarbons," Chemical Physics Letters, vol. 17, no. 4, pp. 535-538, 1972.

[16] M. Eliasi and B. Taeri, "Four new sums of graphs and their Wiener indices," Discrete Applied Mathematics, vol. 157, no. 4, pp. 794-803, 2009.

[17] H. Deng, D. Sarala, S. K. Ayyaswamy, and S. Balachandran, "The Zagreb indices of four operations on graphs," Applied Mathematics and Computation, vol. 275, pp. 422-431, 2016.

[18] S. Akhter and M. Imran, "Computing the forgotten topological index of four operations on graphs," AKCE International Journal of Graphs and Combinatorics, vol. 14, no. 1, pp. 70-79, 2017.

[19] J.-B. Liu, S. Javed, M. Javaid, and K. Shabbir, "Computing first general Zagreb index of operations on graphs," IEEE Access, vol. 7, pp. 47494-47502, 2019.

[20] J.-B. Liu, M. Javaid, and H. M. Awais, "Computing Zagreb indices of the subdivision-related generalized opeations of graphs," IEEE Access, vol. 7, pp. 105479-105488, 2019. 